\title{
Study of Hopf curves in the time delayed active control of a 2DOF nonlinear dynamical system
}

\author{
Ali Kandil ${ }^{1}$
}

Received: 27 July 2020 / Accepted: 30 September 2020 / Published online: 29 October 2020

(c) Springer Nature Switzerland AG 2020

\begin{abstract}
Within this paper, we are focusing on the time delay effects on the improved positive position feedback (PPF) controller for the oscillations of a 2DOF nonlinear dynamical system which represents a thin-walled pre-twisted rotating blade. Herein, we are improving the performance of the traditional positive position feedback controller (PPF) that suffers from the existence of two high amplitude peaks on both sides of its minimum amplitude point. The improvement involves coupling double nonlinear saturation controllers to the main system to notch down the high peaks. As an active control process, the time delay is inherent in the process which produces a locus of Hopf bifurcation points separating between the stable and unstable regions of operation. Variation of different parameters is studied to relate their effects with time delay on the system operation. To ensure the validity of our proposed work, a comparison between the approached multiple scales analytical solutions and the computed Rung-Kutta numerical solutions is done at the end of this paper.
\end{abstract}

Keywords Positive position feedback controller - Nonlinear saturation controller - Multiple time scales method - Time delay · Hopf curves

\section{List of symbols}

$\ddot{p}, \dot{p}, p$

$\ddot{q}, \dot{q}, q$

$\ddot{x}, \dot{x}, x$

$\ddot{y}, \dot{y}, y$

$\ddot{z}, \dot{z}, z$

$\mu, \mu_{1}, \mu_{2}, \mu_{3}$

$\omega, \omega_{1}, \omega_{2}, \omega_{3}$
Horizontal acceleration, velocity and displacement of the blade cross-section

Vertical acceleration, velocity and displacement of the blade cross-section

Acceleration, velocity and displacement of the PPF controller Acceleration, velocity and displacement of the first NSC controller Acceleration, velocity and displacement of the second NSC controller Damping parameters of the blade and controllers Natural frequencies of the blade and controllers

$\begin{array}{ll}\beta_{11}, \beta_{21}, \beta_{13}, \beta_{25}, \beta_{5} & \begin{array}{l}\text { Coupling factors between the blade } \\ \text { vibrational directions } \\ \beta_{5}\end{array} \\ \begin{array}{l}\text { Cubic nonlinearity parameter of the } \\ \text { blade }\end{array} \\ \beta_{14}, \beta_{24} & \begin{array}{l}\text { Parametric excitation parameters } \\ f_{0}, f\end{array} \\ \Omega & \text { Force excitation amplitudes } \\ c_{1}, c_{2} & \text { Excitation frequency } \\ c_{3}, c_{4} & \text { Gains of control signals } \\ \sigma_{1}, \sigma_{2}, \sigma_{3}, \sigma_{4} & \text { Gains of feedback signals } \\ \tau_{1}, \tau_{2} & \text { Detuning parameters }\end{array}$

\section{Introduction}

Thin-walled pre-twisted blades are of the most important structures to be controlled in the modern time due to their participation in many industrial applications. They

Ali Kandil, alikandil21@el-eng.menofia.edu.eg; alikandil21@yahoo.com | Department of Physics and Engineering Mathematics, Faculty of Electronic Engineering, Menoufia University, Menouf 32952, Egypt. 
are built-in components in industrial applications like helicopter blades, robot manipulators and rotating compressor blades that are our case of study in this work. Rotating compressor blades may suffer from large amplitude vibrations that may result in disastrous results, especially at a huge centrifugal force. A lot of researchers around the world tried to model these oscillations to realize how they are destructive and the parameters of dominating that kind of problem. Yoo et al. [1] derived the dimensionless motion equations for the vibration analysis of a pre-twisted, arbitrarily-oriented rotating blade having a concentrated mass in an arbitrary location. They studied the effects of different parameters on the rotating blade dynamics numerically. Sinha [2] illustrated how the rubbing against the rigid outer case in a rotating machinery affects the dynamical behavior of the blades. He concluded that the actual contact load will always be lower than the one computed in his paper for the elastic condition. Fazelzadeh et al. [3] concluded that the application of the differential quadrature method (DQM) for investigating the vibrations of aerothermoelastic thin-walled blades made of functionally graded materials is an efficient numerical tool. Yao et al. [4, 5] used the isotropic constitutive law, Hamilton's principle, and Galerkin's approach to obtain a 2DOF nonlinear dynamical system of a rotating blade with varying speed. Based upon the primary resonance and 1:1 internal resonance cases, they deduced that the periodic motions and chaotic motions can occur in the rotating blade. For eliminating or suppressing the large amplitude oscillations and chaotic motion, theoretical results can help optimize the design of the structural parameters of the rotating thin-walled blade or can help adjust the engine speed to avoid the region of chaotic motion. Younesian and Esmailzadeh [6] suppressed the oscillation of a rotating beam to half of its original vibratory level by proposing the application of a time-increasing internal tensile force. They noticed that constant tensile forces have no effect on vibration reduction even if they have large values. Park and Kim [7] studied the active twisting performance of the active twist rotor (ATR) blade using single crystal macro fiber composite (MFC) which has an excellent twisting actuation performance. Choi et al. [8,9] applied a negative velocity feedback control for realizing a beam's adaptive capability. They used the finite element analysis to show that MFC actuators and Polyvinylidene fluoride (PVDF) sensors could actively control the beam. Vadiraja and Sahasrabudhe [10] emphasized the effects of the pre-twist angle on free or forced vibration characteristics, and controlled response of rotating composite beams. They also concluded that the taper in beam could enhance the first two natural frequencies of the system. Other active control techniques have been applied to nonlinear dynamical systems and were very useful in reducing the vibrations. One of the remarkable active controllers is the positive position feedback controller (PPF). It is a second order differential equation whose input is the position measured feedback of the main system. The output control signal of this controller is applied positively to the main system so it has its name and it must be coupled linearly to the main system. The authors $[11,12]$ analyzed the use of PPF algorithm to suppress the vibrations of a nonlinear composite cantilever beam. They assured that the controller produced high effectiveness at narrow bandwidth of control and that was a major drawback of it. The authors [11, 13 and 14] studied another active vibration control algorithm which is the nonlinear saturation controller (NSC) which is a second order differential equation coupled quadratically to the main system for enhancing the saturation phenomenon. They showed that this controller produced a $\mathrm{V}$-shaped curve to notch the system amplitude down and it gave a quite high effectiveness compared to PPF. Unfortunately, active control techniques have exhibited time delay resulted from measuring the system states, transport delay, on-line computation, filtering and processing of data, calculating and executing of control forces as required in control processing and this is considered a source of instability. Many researchers have studied the stability issue and the performance of delayed control systems. Zhao and Xu [15] studied the application of a feedback control inherently having a time delay to mitigate the vibration of vertical displacement in a 2DOF nonlinear dynamical system that is excited externally. They named a region, where the time delay varied for a fixed gain, for reducing the vibrations safely at some values of the delay as vibration suppression region. Eissa et al. [16] reduced the vibrations in a magnetic levitation system via a time-delayed NSC controller under the excitation of multi forces. They extracted the time delay criterion for producing stable solutions only and for a safer operation. El-Ganaini et al. [17] studied a time-delayed PPF controller in reducing the oscillations of a nonlinear dynamical system. They concluded a relation between time delays to give a border line separating stable from unstable solutions. Kandil and Eissa [18] improved the use of PPF controller in the ideal case without time delay effects. They proposed imposing V-curves at the PPF dual peaks by using two NSC controllers with adjustable natural frequencies to fit for the positions of the peak. Kandil and El-Ganaini [19] investigated the influence of time delay on the stability and efficiency of the PPF controller. The authors illustrated that the loop delay should be kept within specific range to guarantee both the control loop stability and the controller high efficiency.

In this paper, we are going to mix the jobs of both controllers PPF and NSC and observe the time delay effect on the whole system after control for the reported model [4, 
5]. Due to the strong coupling between the blade horizontal and vertical directions, the controllers are applied to the first mode only and the second mode follows it. The multiple time scales method is conducted to derive the steady state equations and the results are verified by numerical simulations. The paper is organized in six sections. Section 1 introduced a short concept about the rotating blade and the previously published work in the same field. Section 2 provides the controlled blade's equations of motion and the approximate solutions to such equations. Section 3 includes response curves of the system based upon the extracted approximate solutions. Section 4 shows a numerical simulation of the blade vibrations before and after control. Section 5 shows a comparison between the solutions gained by the multiple scales method and the ones gained by numerical simulations. The conclusions on the proposed study are in Sect. 6.

\section{System model and multiple time scales analysis}

The horizontal and vertical deflections of the blade's crosssection have been described by $u_{0}$ and $v_{0}$ in $[4,5]$. To ease studying the blade dynamics, the authors have applied the idea of one-term Galerkin's approach to truncate the system governing partial differential equations to obtain a 2DOF nonlinear system of ordinary differential equations in terms of the temporal deflections $p(t)$ and $q(t)$, the fundamental modes of $u_{0}$ and $v_{0}$, respectively. A brief derivation is given in "Appendix A". So, the blade's cross-section equations of motion subjected to a periodic excitation are expressed as follows:

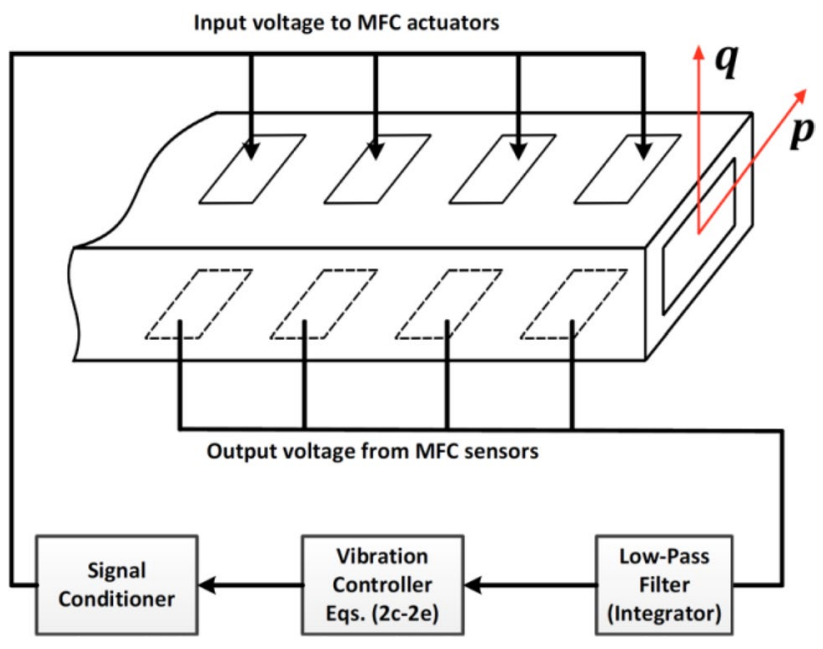

Fig. 1 Process of sensing and actuating from and into MFC

produce a voltage proportional to the derivative of the blade horizontal deflection $\dot{p}(t)$ as in Refs.[7-9]. After that, it is passed through a low-pass filter which acts as an integrator to produce the feedback signal $p(t)$ inserted to the vibration controller unit as Ref. [10]. Our proposed controller awaits this signal for processing it and produces a control signal $F_{c}(t)$. This control signal needs to be conditioned through a signal conditioning circuit for providing the MFC actuators with the suitable voltage as stated in Refs .[7-10]. The embedded MFC sensors are distributed over the bottom surface of the blade while the embedded MFC actuators are distributed over the top of the blade as shown in Fig. 1. The whole operation continues until the steady state behavior becomes small compared to the one before control. The classical PPF controller is a virtual

$\ddot{p}+2 \mu \dot{p}+\omega^{2} p+\beta_{13} \dot{q}+\beta_{11} q+\beta_{5} p q^{2}+\beta_{5} p^{3}=2 f_{0} f \beta_{14} p \cos (\Omega t)+f^{2} \beta_{14} p \cos ^{2}(\Omega t)+f \beta_{16} \Omega \sin (\Omega t)$

$\ddot{q}+2 \mu \dot{q}+\omega^{2} q+\beta_{22} \dot{p}+\beta_{21} p+\beta_{5} p^{2} q+\beta_{5} q^{3}=2 f_{0} f \beta_{24} q \cos (\Omega t)+f^{2} \beta_{24} q \cos ^{2}(\Omega t)$

We can see that Eq. 1.a is the only one excited by external excitation $f \beta_{16} \Omega \sin (\Omega t)$ which is very effective in the primary resonance case. Hence, we should apply the control signal only to Eq. 1.a and then Eq. 1.b will follow it due to the strong coupling between the two equations thanks to the inherent internal resonance 1:1. We can add the control forces $F_{c}(t)$ as was stated before in Refs. [7-10]. The improved PPF controller can be programmed in a computerized control unit which produces a control signal to be applied on the actuator as shown in Fig. 1 .

When the piezoelectric sensors are used as strain rate sensors, the current can be converted into sensor voltage output [7-9]. When the blade oscillates, the MFC sensors second order oscillator that is programmed on a microchip and is coupled linearly to the main vibratory system to be controlled. Its major drawback is creating high-amplitude peaks besides the control region. An improvement can be done by coupling an NSC controller at each peak for notching down that peak to its lowest level. Moreover, the sensing and actuating mechanisms through the microchip may be delayed. This problem has been taken into account to study its effects on the whole operation. After coupling the improved PPF controller to the main system (Eq. 1) considering the time delays, the modified controlled equations of motion will be: 


$$
\begin{gathered}
\ddot{p}+2 \mu \dot{p}+\omega^{2} p+\beta_{13} \dot{q}+\beta_{11} q+\beta_{5} p q^{2}+\beta_{5} p^{3} \\
=2 f_{0} f \beta_{14} p \cos (\Omega t)+f^{2} \beta_{14} p \cos ^{2}(\Omega t) \\
+f \beta_{16} \Omega \sin (\Omega t)+c_{1} x\left(t-\tau_{2}\right) \\
+c_{2}\left[y^{2}\left(t-\tau_{2}\right)+z^{2}\left(t-\tau_{2}\right)\right] \\
\ddot{q}+2 \mu \dot{q}+\omega^{2} q+\beta_{22} \dot{p}+\beta_{21} p+\beta_{5} p^{2} q+\beta_{5} q^{3} \\
=2 f_{0} f \beta_{24} q \cos (\Omega t)+f^{2} \beta_{24} q \cos ^{2}(\Omega t) \\
\ddot{x}+2 \mu_{1} \dot{x}+\omega_{1}^{2} x=c_{3} p\left(t-\tau_{1}\right) \\
\ddot{y}+2 \mu_{2} \dot{y}+\omega_{2}^{2} y=c_{4} y p\left(t-\tau_{1}\right) \\
\ddot{z}+2 \mu_{3} \dot{z}+\omega_{3}^{2} z=c_{4} z p\left(t-\tau_{1}\right)
\end{gathered}
$$

The parameters are suitably scaled such that:

$$
\begin{aligned}
& \mu=\varepsilon \hat{\mu}, \mu_{1,2,3}=\varepsilon \hat{\mu}_{1,2,3}, \beta_{11}=\varepsilon \hat{\beta}_{11}, \beta_{13}=\varepsilon \hat{\beta}_{13} \\
& \beta_{14}=\varepsilon \hat{\beta}_{14}, \beta_{16}=\varepsilon \hat{\beta}_{16}, \beta_{21}=\varepsilon \hat{\beta} 2_{21} \beta_{22}=\varepsilon \hat{\beta} 2_{22} \\
& \beta_{24}=\varepsilon \hat{\beta}_{24}, \beta_{5}=\varepsilon \hat{\beta}_{5}, c_{1,2,3,4}=\varepsilon \hat{c}_{1,2,3,4}
\end{aligned}
$$

where $\varepsilon$ is a small perturbation parameter. Applying the multiple time scales method [20], the asymptotic expansions of approximate solutions are sought as:

$$
\begin{aligned}
& p(t ; \varepsilon)=p_{0}\left(T_{0}, T_{1}\right)+\varepsilon p_{1}\left(T_{0}, T_{1}\right)+O\left(\varepsilon^{2}\right) \\
& q(t ; \varepsilon)=q_{0}\left(T_{0}, T_{1}\right)+\varepsilon q_{1}\left(T_{0}, T_{1}\right)+O\left(\varepsilon^{2}\right) \\
& x(t ; \varepsilon)=x_{0}\left(T_{0}, T_{1}\right)+\varepsilon x_{1}\left(T_{0}, T_{1}\right)+O\left(\varepsilon^{2}\right) \\
& y(t ; \varepsilon)=y_{0}\left(T_{0}, T_{1}\right)+\varepsilon y_{1}\left(T_{0}, T_{1}\right)+O\left(\varepsilon^{2}\right) \\
& z(t ; \varepsilon)=z_{0}\left(T_{0}, T_{1}\right)+\varepsilon z_{1}\left(T_{0}, T_{1}\right)+O\left(\varepsilon^{2}\right) \\
& p\left(t-\tau_{1} ; \varepsilon\right)=p_{0 \tau_{1}}\left(T_{0}, T_{1}\right)+\varepsilon p_{1 \tau_{1}}\left(T_{0}, T_{1}\right)+O\left(\varepsilon^{2}\right) \\
& x\left(t-\tau_{2} ; \varepsilon\right)=x_{0 \tau_{2}}\left(T_{0}, T_{1}\right)+\varepsilon x_{1 \tau_{2}}\left(T_{0}, T_{1}\right)+O\left(\varepsilon^{2}\right) \\
& y\left(t-\tau_{2} ; \varepsilon\right)=y_{0 \tau_{2}}\left(T_{0}, T_{1}\right)+\varepsilon y_{1 \tau_{2}}\left(T_{0}, T_{1}\right)+O\left(\varepsilon^{2}\right) \\
& z\left(t-\tau_{2} ; \varepsilon\right)=z_{0 \tau_{2}}\left(T_{0}, T_{1}\right)+\varepsilon z_{1 \tau_{2}}\left(T_{0}, T_{1}\right)+O\left(\varepsilon^{2}\right)
\end{aligned}
$$

where $T_{0}=t, T_{1}=\varepsilon t$ are the time scales. The time derivatives will be converted to: $\frac{d}{d t}=D_{0}+\varepsilon D_{1}+O\left(\varepsilon^{2}\right)$

$\frac{d^{2}}{d t^{2}}=D_{0}^{2}+2 \varepsilon D_{1} D_{0}+O\left(\varepsilon^{2}\right)$

where $D_{i}=\partial / \partial T_{i}, i=0$, 1. The deduced simultaneous resonance case to be studied in this work is $\Omega \cong \omega, \omega_{1} \cong \omega$, $2 \omega_{2} \cong \omega$, and $2 \omega_{3} \cong \omega$ where the closeness between these different frequencies are expressed by the detuning parameters $\sigma_{1}, \sigma_{2}, \sigma_{3}$, and $\sigma_{4}$, respectively. Substituting Eq. 3-5 into Eq. 2 then following the procedure of the multiple scales technique [20] ("Appendix B") to find the following autonomous system of differential equations:

$$
\begin{aligned}
a_{1}= & -\mu a_{1}-\frac{\beta_{13}}{2} a_{2} \cos \phi_{2}-\frac{\beta_{11}}{2 \omega} a_{2} \sin \phi_{2}-\frac{\beta_{5}}{8 \omega} a_{1} a_{2}^{2} \sin \left(2 \phi_{2}\right) \\
& +\frac{\beta_{14} f^{2}}{8 \omega} a_{1} \sin \left(2 \phi_{1}\right)-\frac{\beta_{16} \Omega f}{2 \omega} \cos \phi_{1}+\frac{c_{1}}{2 \omega} a_{3} \sin \left(\phi_{3}-\omega_{1} \tau_{2}\right) \\
& +\frac{c_{2}}{4 \omega} a_{4}^{2} \sin \left(\phi_{4}-2 \omega_{2} \tau_{2}\right)+\frac{c_{2}}{4 \omega} a_{5}^{2} \sin \left(\phi_{5}-2 \omega_{3} \tau_{2}\right) \\
\dot{\phi}_{1}= & \sigma_{1}+\frac{\beta_{13}}{2} \frac{a_{2}}{a_{1}} \sin \phi_{2}-\frac{\beta_{11}}{2 \omega} \frac{a_{2}}{a_{1}} \cos \phi_{2}-\frac{\beta_{5}}{4 \omega} a_{2}^{2} \\
& -\frac{\beta_{5}}{8 \omega} a_{2}^{2} \cos \left(2 \phi_{2}\right)-\frac{3 \beta_{5}}{8 \omega} a_{1}^{2}+\frac{\beta_{14} f^{2}}{8 \omega} \cos \left(2 \phi_{1}\right) \\
& +\frac{\beta_{14} f^{2}}{4 \omega}+\frac{\beta_{16} \Omega f}{2 \omega} \frac{1}{a_{1}} \sin \phi_{1}+\frac{c_{1}}{2 \omega} \frac{a_{3}}{a_{1}} \cos \left(\phi_{3}-\omega_{1} \tau_{2}\right) \\
& +\frac{c_{2}}{4 \omega} \frac{a_{4}^{2}}{a_{1}} \cos \left(\phi_{4}-2 \omega_{2} \tau_{2}\right)+\frac{c_{2}}{4 \omega} \frac{a_{5}^{2}}{a_{1}} \cos \left(\phi_{5}-2 \omega_{3} \tau_{2}\right)
\end{aligned}
$$

$$
\begin{aligned}
\dot{a}_{2}= & -\mu a_{2}-\frac{\beta_{22}}{2} a_{1} \cos \phi_{2}+\frac{\beta_{5}}{8 \omega} a_{1}^{2} a_{2} \sin \left(2 \phi_{2}\right) \\
& +\frac{\beta_{24} f^{2}}{8 \omega} a_{2} \sin \left(2 \phi_{1}-2 \phi_{2}\right)+\frac{\beta_{21}}{2 \omega} a_{1} \sin \phi_{2}
\end{aligned}
$$

$$
\begin{aligned}
\dot{\phi}_{2}= & \frac{\beta_{22}}{2} \frac{a_{1}}{a_{2}} \sin \phi_{2}+\frac{\beta_{21}}{2 \omega} \frac{a_{1}}{a_{2}} \cos \phi_{2}+\frac{\beta_{5}}{4 \omega} a_{1}^{2} \\
& +\frac{\beta_{5}}{8 \omega} a_{1}^{2} \cos \left(2 \phi_{2}\right)+\frac{3 \beta_{5}}{8 \omega} a_{2}^{2}-\frac{\beta_{24} f^{2}}{8 \omega} \cos \left(2 \phi_{1}-2 \phi_{2}\right) \\
& -\frac{\beta_{24} f^{2}}{4 \omega}+\frac{\beta_{13}}{2} \frac{a_{2}}{a_{1}} \sin \phi_{2}-\frac{\beta_{11}}{2 \omega} \frac{a_{2}}{a_{1}} \cos \phi_{2} \\
& -\frac{\beta_{5}}{4 \omega} a_{2}^{2}-\frac{\beta_{5}}{8 \omega} a_{2}^{2} \cos \left(2 \phi_{2}\right)-\frac{3 \beta_{5}}{8 \omega} a_{1}^{2} \\
& +\frac{\beta_{14} f^{2}}{8 \omega} \cos \left(2 \phi_{1}\right)+\frac{\beta_{14} f^{2}}{4 \omega}+\frac{\beta_{16} \Omega f}{2 \omega} \frac{1}{a_{1}} \sin \phi_{1} \\
& +\frac{c_{1}}{2 \omega} \frac{a_{3}}{a_{1}} \cos \left(\phi_{3}-\omega_{1} \tau_{2}\right)+\frac{c_{2}}{4 \omega} \frac{a_{4}^{2}}{a_{1}} \cos \left(\phi_{4}-2 \omega_{2} \tau_{2}\right) \\
& +\frac{c_{2}}{4 \omega} \frac{a_{5}^{2}}{a_{1}} \cos \left(\phi_{5}-2 \omega_{3} \tau_{2}\right)
\end{aligned}
$$




$$
\begin{aligned}
\dot{a}_{3}= & -\mu_{1} a_{3}-\frac{c_{3}}{2 \omega_{1}} a_{1} \sin \left(\phi_{3}+\omega \tau_{1}\right) \\
\dot{\phi}_{3}= & \sigma_{2}-\frac{c_{3}}{2 \omega_{1}} \frac{a_{1}}{a_{3}} \cos \left(\phi_{3}+\omega \tau_{1}\right)+\frac{\beta_{13}}{2} \frac{a_{2}}{a_{1}} \sin \phi_{2} \\
& -\frac{\beta_{11}}{2 \omega} \frac{a_{2}}{a_{1}} \cos \phi_{2}-\frac{\beta_{5}}{4 \omega} a_{2}^{2}-\frac{\beta_{5}}{8 \omega} a_{2}^{2} \cos \left(2 \phi_{2}\right) \\
& -\frac{3 \beta_{5}}{8 \omega} a_{1}^{2}+\frac{\beta_{14} f^{2}}{8 \omega} \cos \left(2 \phi_{1}\right)+\frac{\beta_{16} \Omega f}{2 \omega} \frac{1}{a_{1}} \sin \phi_{1} \\
& +\frac{\beta_{14} f^{2}}{4 \omega}+\frac{c_{1}}{2 \omega} \frac{a_{3}}{a_{1}} \cos \left(\phi_{3}-\omega_{1} \tau_{2}\right) \\
& +\frac{c_{2}}{4 \omega} \frac{a_{4}^{2}}{a_{1}} \cos \left(\phi_{4}-2 \omega_{2} \tau_{2}\right)
\end{aligned}
$$

$$
\dot{a}_{4}=-\mu_{2} a_{4}-\frac{c_{4}}{4 \omega_{2}} a_{1} a_{4} \sin \left(\phi_{4}+\omega \tau_{1}\right)
$$

$$
\begin{aligned}
\dot{\phi}_{4}= & \sigma_{3}-\frac{c_{4}}{2 \omega_{2}} a_{1} \cos \left(\phi_{4}+\omega \tau_{1}\right)+\frac{\beta_{13}}{2} \frac{a_{2}}{a_{1}} \sin \phi_{2}-\frac{\beta_{11}}{2 \omega} \frac{a_{2}}{a_{1}} \cos \phi_{2} \\
& -\frac{\beta_{5}}{4 \omega} a_{2}^{2}-\frac{\beta_{5}}{8 \omega} a_{2}^{2} \cos \left(2 \phi_{2}\right)-\frac{3 \beta_{5}}{8 \omega} a_{1}^{2}+\frac{\beta_{14} f^{2}}{4 \omega}+\frac{\beta_{14} f^{2}}{8 \omega} \cos \left(2 \phi_{1}\right) \\
& +\frac{\beta_{16} \Omega f}{2 \omega} \frac{1}{a_{1}} \sin \phi_{1}+\frac{c_{1}}{2 \omega} \frac{a_{3}}{a_{1}} \cos \left(\phi_{3}-\omega_{1} \tau_{2}\right) \\
& +\frac{c_{2}}{4 \omega} \frac{a_{4}^{2}}{a_{1}} \cos \left(\phi_{4}-2 \omega_{2} \tau_{2}\right)+\frac{c_{2}}{4 \omega} \frac{a_{5}^{2}}{a_{1}} \cos \left(\phi_{5}-2 \omega_{3} \tau_{2}\right)
\end{aligned}
$$$$
\dot{a}_{5}=-\mu_{3} a_{5}-\frac{c_{4}}{4 \omega_{3}} a_{1} a_{5} \sin \left(\phi_{5}+\omega \tau_{1}\right)
$$$$
\dot{\phi}_{5}=\sigma_{4}-\frac{c_{4}}{2 \omega_{3}} a_{1} \cos \left(\phi_{5}+\omega \tau_{1}\right)+\frac{\beta_{13}}{2} \frac{a_{2}}{a_{1}} \sin \phi_{2}
$$$$
-\frac{\beta_{11}}{2 \omega} \frac{a_{2}}{a_{1}} \cos \phi_{2}-\frac{\beta_{5}}{4 \omega} a_{2}^{2}-\frac{\beta_{5}}{8 \omega} a_{2}^{2} \cos \left(2 \phi_{2}\right)
$$$$
-\frac{3 \beta_{5}}{8 \omega} a_{1}^{2} \dot{\phi}_{5}+\frac{\beta_{14} f^{2}}{4 \omega}+\frac{\beta_{14} f^{2}}{8 \omega} \cos \left(2 \phi_{1}\right)
$$$$
+\frac{\beta_{16} \Omega f}{2 \omega} \frac{1}{a_{1}} \sin \phi_{1}+\frac{c_{1}}{2 \omega} \frac{a_{3}}{a_{1}} \cos \left(\phi_{3}-\omega_{1} \tau_{2}\right)
$$$$
+\frac{c_{2}}{4 \omega} \frac{a_{4}^{2}}{a_{1}} \cos \left(\phi_{4}-2 \omega_{2} \tau_{2}\right)+\frac{c_{2}}{4 \omega} \frac{a_{5}^{2}}{a_{1}} \cos \left(\phi_{5}-2 \omega_{3} \tau_{2}\right)
$$

where $a_{n}, \phi_{n}(n=1,2, \ldots, 5)$ are the amplitudes and phases of the blade and controllers. To get the steady state equations that describe the modulating amplitudes and phases of the blade and the controllers, we should put $\dot{a}_{n}=\dot{\phi}_{n}=0(n=1,2, \cdots, 5)$ into Eq. 6 . Since there is no closed form solution of the resulting nonlinear algebraic equations system, we turn to numerical techniques in solving such a system. Also, the stability of these solutions is analyzed by resorting to the first (indirect) method of Lyapunov as follows:

$$
\left(\begin{array}{c}
\dot{a}_{1} \\
\dot{\phi}_{1} \\
\dot{a}_{2} \\
\dot{\phi}_{2} \\
\dot{a}_{3} \\
\dot{\phi}_{3} \\
\dot{a}_{4} \\
\dot{\phi}_{4} \\
\dot{a}_{5} \\
\dot{\phi}_{5}
\end{array}\right)=\left[J_{i j}\right]_{10 \times 10}\left(\begin{array}{c}
a_{1} \\
\phi_{1} \\
a_{2} \\
\phi_{2} \\
a_{3} \\
\phi_{3} \\
a_{4} \\
\phi_{4} \\
a_{5} \\
\phi_{5}
\end{array}\right)
$$

where $J_{i j}(i, j=1,2, \cdots, 10)$ are the entries of Jacobian matrix that are included in "Appendix C". If and only if all the real parts of the Jacobian matrix's eigenvalues are negative, then the steady state solution is asymptotically stable and otherwise, it is unstable.

\section{Results and discussion}

In this section, we are going to show the effects of time delays issue on different responses (frequency, force, time), then a validation to the proposed work is introduced to show the degree of closeness between the perturbation curves and the numerically simulated responses. The importance of this work is represented in giving a recommendation of the safe regions of time delays for a stable and safe operation of the studied rotating blade.

\subsection{Perturbation curves}

All the resulted perturbation equilibrium curves are plotted depending on putting $\dot{a}_{n}=\dot{\phi}_{n}=0(n=1,2, \cdots, 5)$ into Eq. 6 and are discussed in this section. The stable analytical solutions are denoted by solid lines while the unstable ones are denoted by dashed lines. The asterisks denote the bifurcation points (SN stands for SaddleNode, $\mathrm{H}$ stands for Hopf and PF stands for Pitchfork). The overall adopted parameters values are given by: $\mu=0.5, \mu_{1}=0.005, \mu_{2}=\mu_{3}=0.001, \Omega=\omega=\omega_{1}=100$, $\omega_{2}=52.5, \omega_{3}=47.65, \beta_{13}=\beta_{22}=-0.82, \beta_{11}=-0.003$, $\beta_{14}=0.55, \beta_{5}=0.9, \beta_{16}=6.55, \beta_{21}=-0.001, \beta_{24}=0.5$, $f_{0}=7, f=2, c_{1}=c_{3}=1000, c_{2}=5, c_{4}=50, \sigma_{1}=\sigma_{2}=0$, $\sigma_{3}=5, \sigma_{4}=-4.7, \tau_{1}=\tau_{2}=0$, unless otherwise specified. Figure $2 \mathrm{a}$ and $\mathrm{b}$ show the relation of the blade horizontal $\left(a_{1}\right)$ and vertical $\left(a_{2}\right)$ deflection steady amplitudes versus the detuning $\sigma_{1}\left(\sigma_{1}=\Omega-\omega\right)$ before control, while Fig. $2 \mathrm{c}$ and $d$ indicate the state planes of blade amplitudes and phases before control. As shown in Fig. $2 a$ and $b$ that the blade suffers from jump phenomena, saddle-node bifurcation and Hopf bifurcation. We have explained how the jump phenomena occurred by drawing colored arrows 

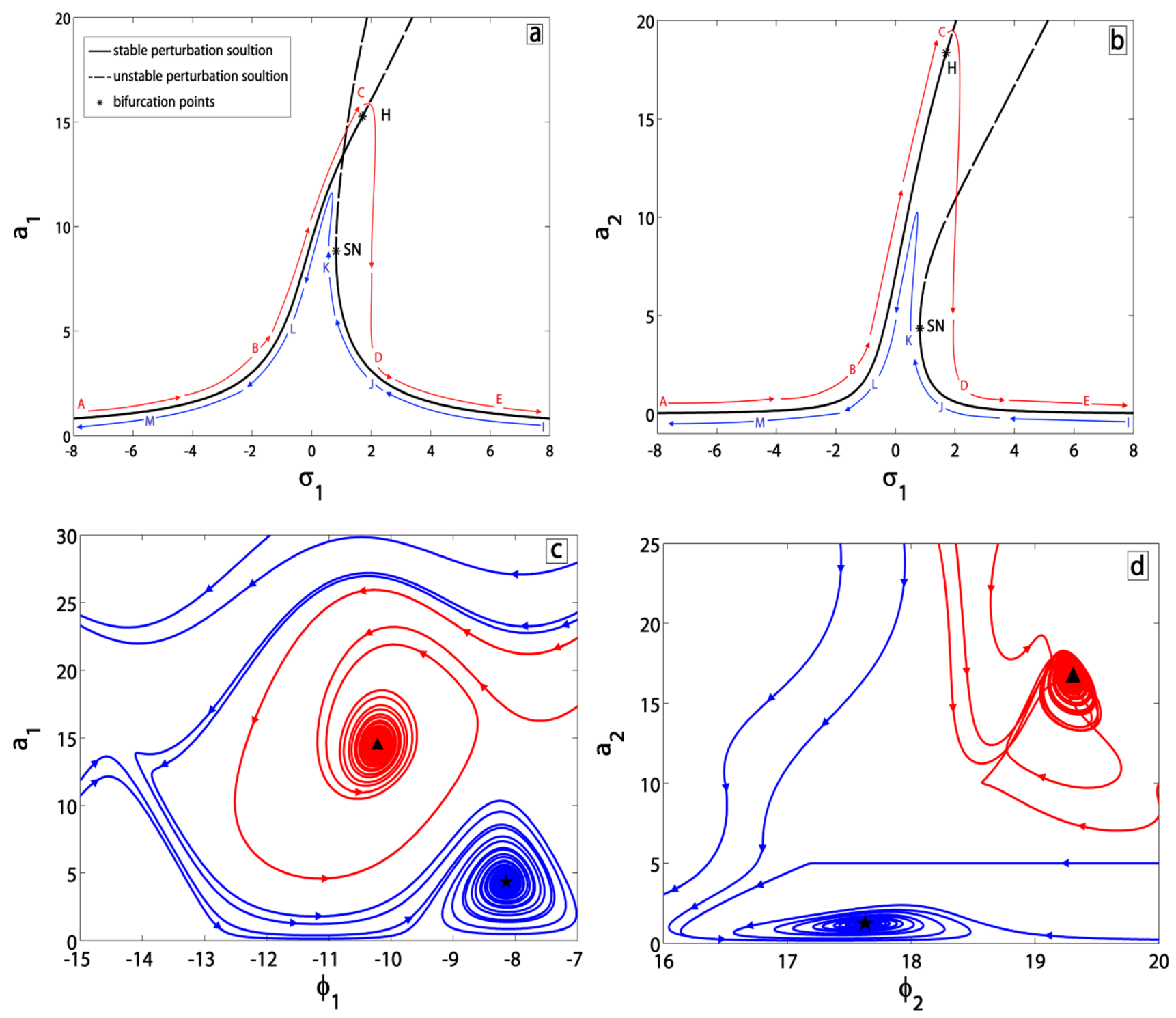

Fig. 2 Blade deflections before control: $\mathbf{a}, \mathbf{b}$ frequency response curves, $\mathbf{C}, \mathbf{d}$ state planes at $\sigma_{1} \cong 1.4$

indicating the operation behavior. During increasing $\sigma_{1}$ (red arrows), the vibration amplitudes leaves point $A$ and increase slightly until they reach point $B$. Then, they begin to increase sharply until point $C$ (Hopf bifurcation point). Sudden jump-down happens to the amplitudes where they decrease drastically to point $D$, and then decrease slightly to reach point $E$. On the other hand when decreasing $\sigma_{1}$ (blue arrows), the amplitudes leave point $I$ and increase slightly reaching $J$. The amplitudes then increase sharply till the point $K$ (saddle-node bifurcation point). The sudden jump-up happens to the amplitudes where they increase then decrease roughly to point $L$, then decrease slightly to reach $M$. Figure $2 \mathrm{c}$ and $\mathrm{d}$ confirm that there are multiple equilibrium points (stable foci) at $\sigma_{1} \cong 1.4$. The higher amplitude focus point is denoted by a black triangle whilst the lower amplitude focus point is denoted by a black star. These two plots assure the existence of jump phenomena between two amplitude values at the same frequency.

The conventional PPF controller had to be splendid at $\sigma_{1}=0$ but it had a problem of two high amplitude peaks on both sides of $\sigma_{1}=0$. Our work is to overcome that problem and it is clear in Fig. 3. Figure 3 demonstrates the same relation shown in Fig. $2 a$ and $b$ but after applying the control algorithm with zero time delays (ideal case $\left.\tau_{1}=\tau_{2}=0\right)$. We can see that the bifurcation points ( $\mathrm{H}$ and SN) and the jump phenomena have been eliminated successfully [18]. In addition, the appearance of pitchfork bifurcation points is not a drawback; it is an advantage to 

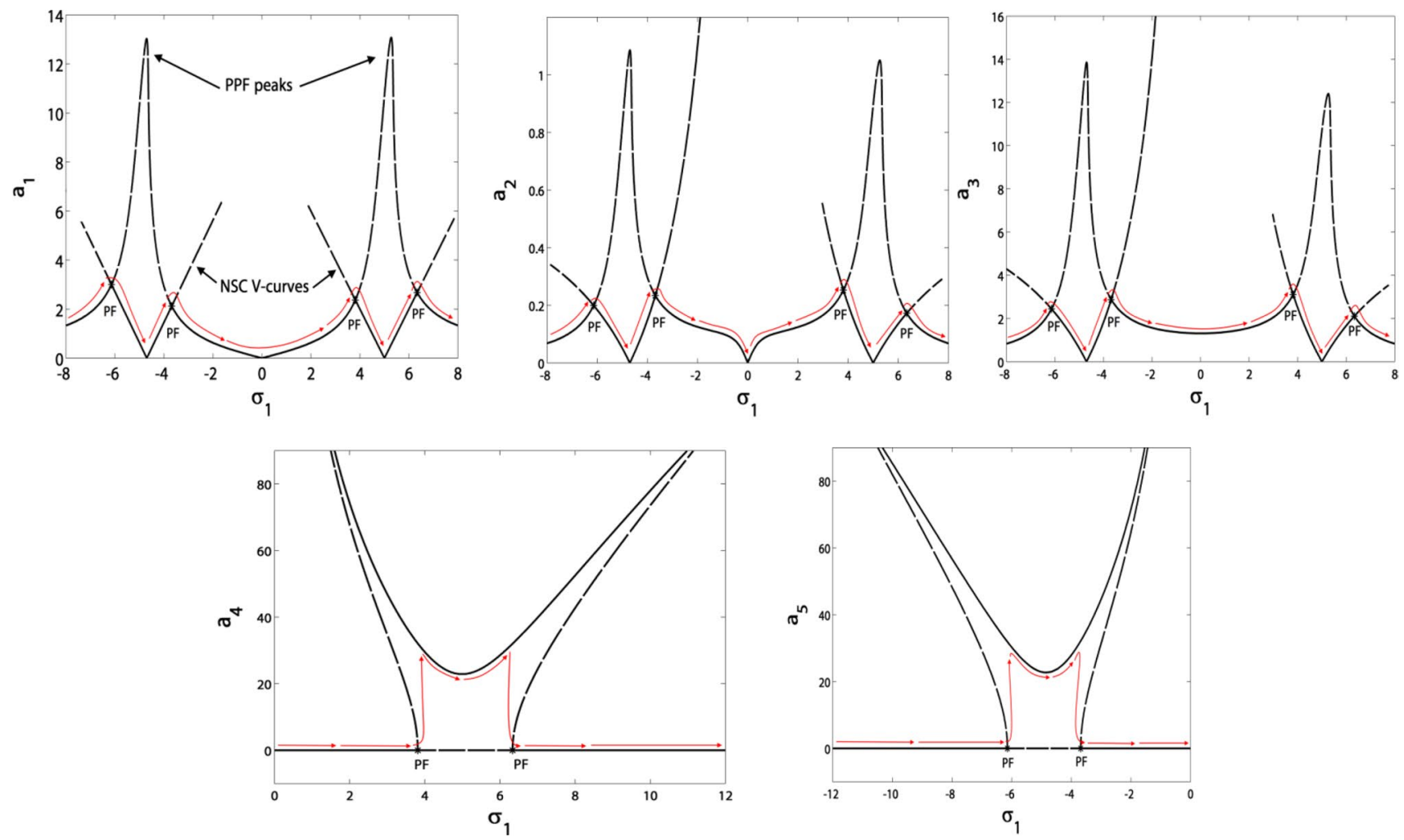

Fig. 3 Blade and controller frequency response curves $\left(\tau_{1}=\tau_{2}=0\right)$

switch the path of the amplitudes as pictured by the red arrows.

It is time to discuss the way of determining the safe region for amplitudes counting on time delays $\tau_{1}, \tau_{2}$ and the detuning parameter $\sigma_{1}:-4.7 \rightarrow+5$. In Fig. 4 , the region of stable amplitudes has been plotted as a function of the detuning parameter $\sigma_{1}$ and the combination $\tau_{1}+\tau_{2}$ at various PPF gains $c_{1}$ and $c_{3}$. The shaded region portrays stable amplitudes while the un-shaded region is for unstable amplitudes. The blue barrier curve between stability and instability regions is the locus of Hopf bifurcation points. Another dashed-dotted vertical line in the shaded region denotes that the left side of it is the safest region for operation. It can be seen from the figure that the region of stable amplitudes shrinks with increasing the gains. In a similar way, Fig. 5 shows the region of stable amplitudes as a function of the detuning parameter $\sigma_{1}$ and the combination $\tau_{1}+\tau_{2}$ at various NSC gains $c_{2}$ and $c_{4}$. We can
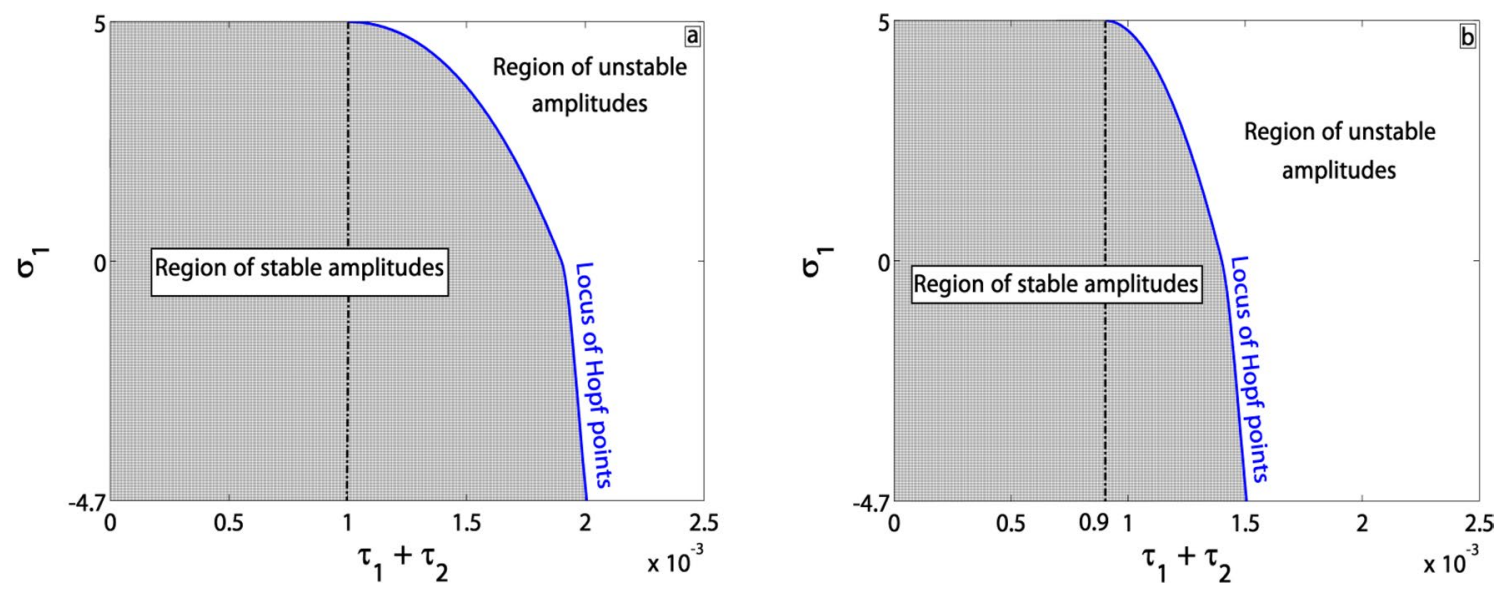

Fig. 4 Stable amplitudes region as a function of $\sigma_{1}$ and $\tau_{1}+\tau_{2}$ : $\mathbf{a}$ at $c_{1}=c_{3}=500, \mathbf{b}$ at $c_{1}=c_{3}=700$ 

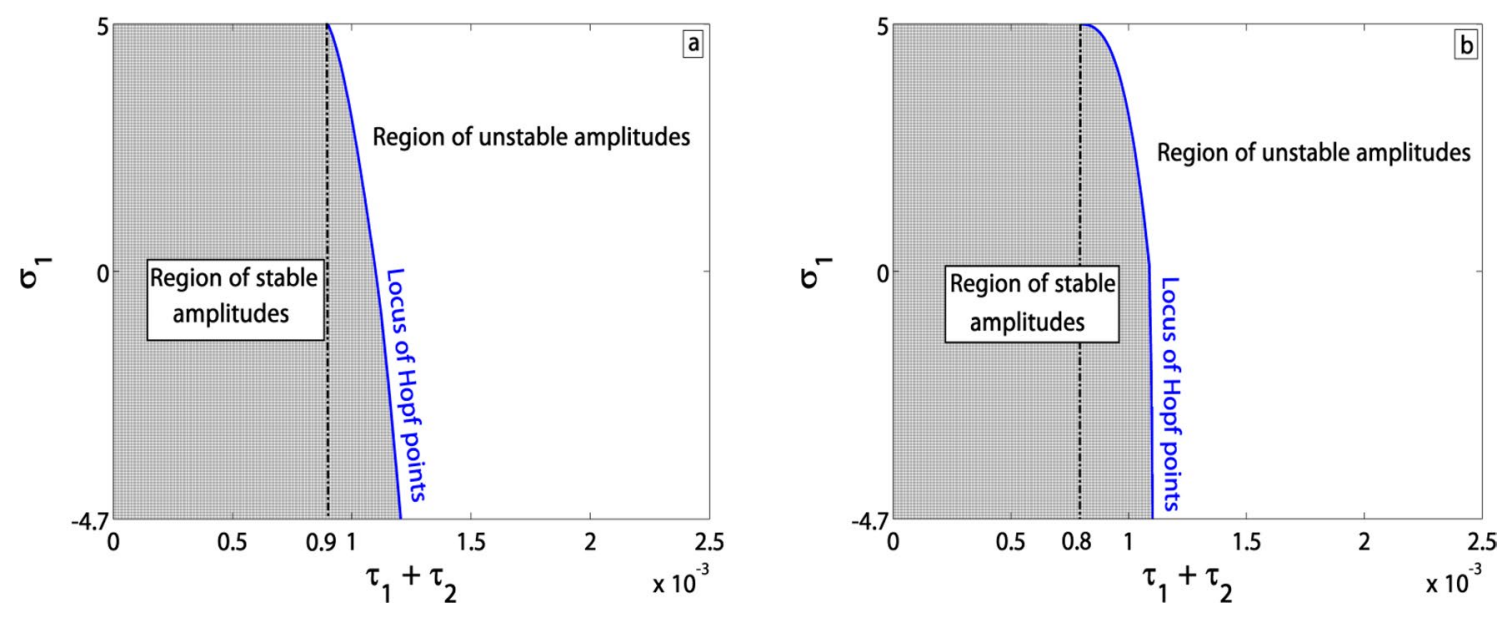

Fig. 5 Stable amplitudes region: $\mathbf{a}$ at $c_{2}=3, c_{4}=15, \mathbf{b}$ at $c_{2}=4, c_{4}=30$

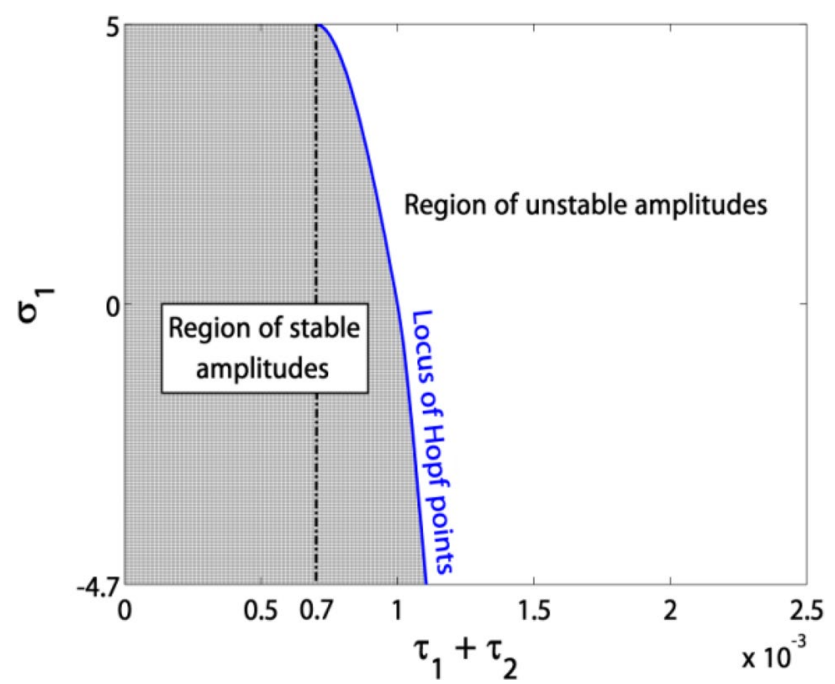

Fig. 6 Stable amplitudes region at $c_{1}=c_{3}=1000, c_{2}=5, c_{4}=50$

note that the region of stable amplitudes slightly shrinks but with a steep barrier curve (locus of Hopf points). Upon Figs. 4 and 5, we can proceed to Fig. 6 where we plotted the same region but at the optimum values of PPF and NSC gains. We can see from the figure that $\tau_{1}+\tau_{2}<0.0011$ is good for producing stable amplitudes but not for all the values of $\sigma_{1}$ in the interval $[-4.7,+5]$. Also, we can see that $\tau_{1}+\tau_{2}<0.0007$ is the ultimate condition for stable amplitudes for all the values of $\sigma_{1}$ in the interval $[-4.7,+5]$. As a consequence, we have plotted the region of stable amplitudes as a function of $\tau_{1}$ and $\tau_{2}$ at the optimum gains and different values of $\sigma_{1}$ in Fig. 7. It assures that increasing $\sigma_{1}$ reduces the safe region of both time delays to tell us that the narrowest shown region is the safest for the values of $\sigma_{1}$ in the range $[-4.7,+5]$.

Figure 8 shows again the blade and controller frequency response curves without time delays (black curves)

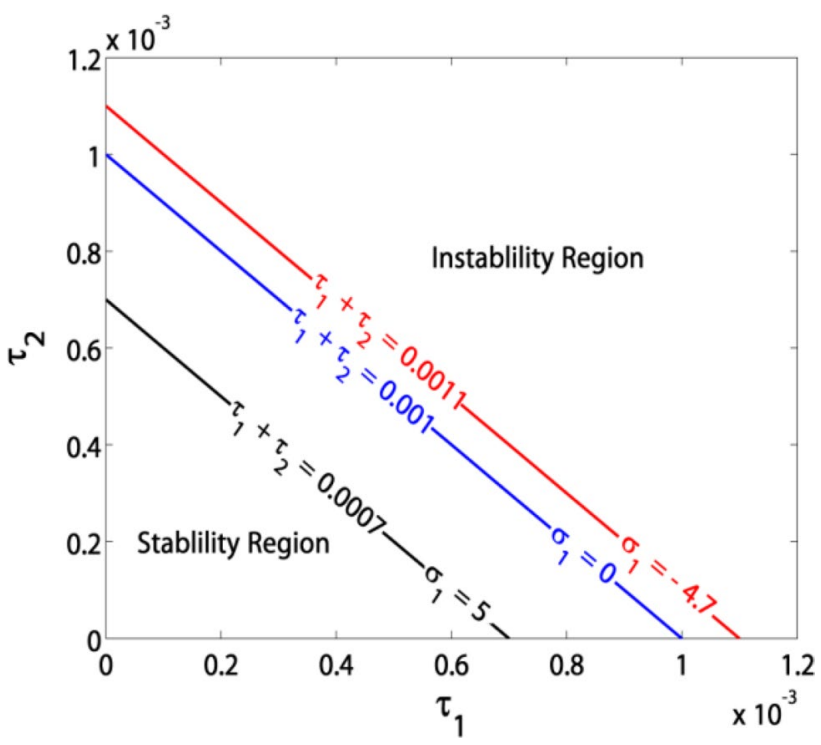

Fig. 7 Stable amplitudes region as a function of $\tau_{1}$ and $\tau_{2}$ at $c_{1}=c_{3}=1000, c_{2}=5, c_{4}=50$

and with time delays (blue curves). For the blade deflection amplitudes $a_{1}, a_{2}$ and the PPF amplitude $a_{3}$, the path of operation (discussed in Fig. 3) has not been affected by the presence of time delay. On the other hand, the NSC amplitudes $a_{4}, a_{5}$ have been slightly changed at their peaks and it does not matter because all we care about are the control points at $\sigma_{1}=\{-4.7,+5\}$ that were not affected at all by the time delay appearance.

Figures 9, 10 and 11 show the force response curves of the blade deflection amplitudes and the controllers amplitudes at different values of $\sigma_{1}$. The responses before control are denoted by blue lines while the controlled responses are denoted by black lines. In all of these figures, it is clear that the slopes of the input-output amplitudes relations 

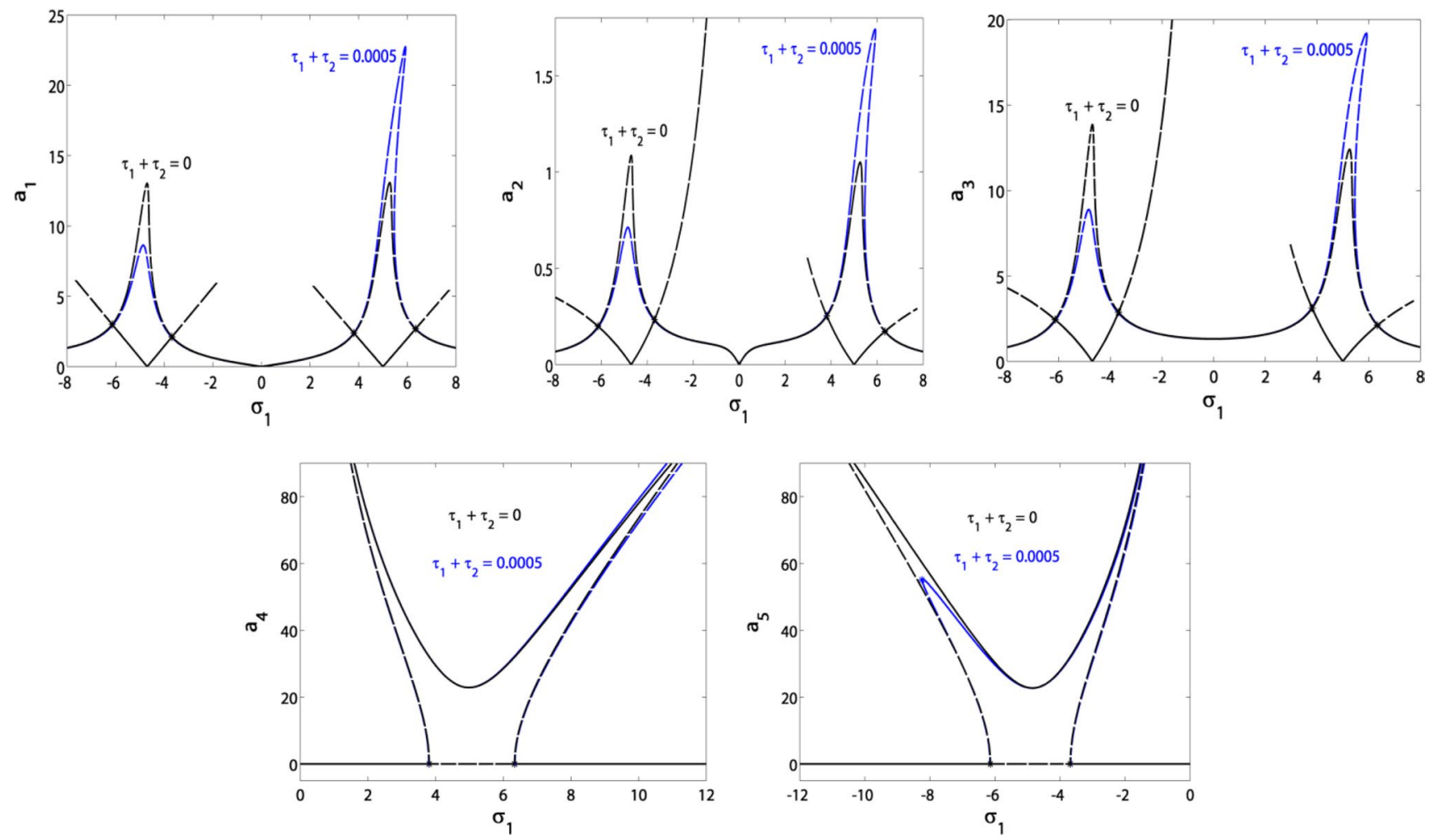

Fig. 8 Blade and controllers frequency response curves at $\tau_{1}+\tau_{2}=0$ (black), and $\tau_{1}+\tau_{2}=0.0005$ (blue)
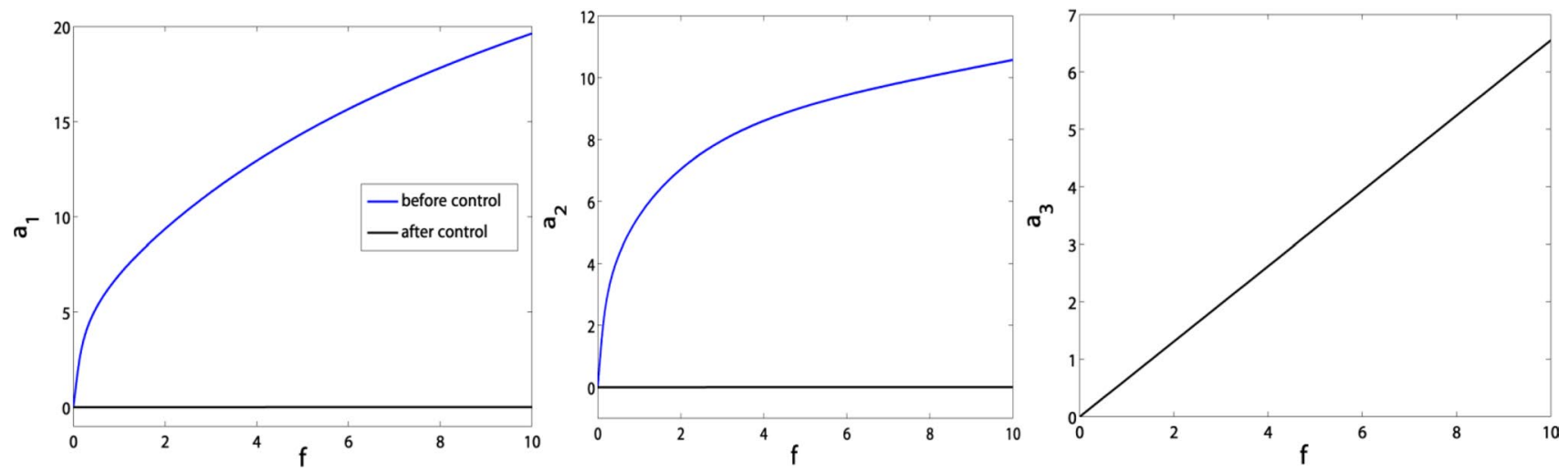

Fig. 9 Force response curves before control (blue) and after control (black) at $\sigma_{1}=0, \tau_{1}+\tau_{2}=0.0005$

have changed after control to become almost horizontal at zero. This is useful for producing very small output amplitudes even if the input excitation forces increased roughly especially at the indicated control points $\sigma_{1}=\{-4.7,0,+5\}$

\subsection{Numerical simulation}

In this section, we solve Eq. 2 numerically via the fourth order Runge-Kutta method which is a built-in algorithm in MATLAB as ODE45 (for ordinary differential equations) and DDE23 (for delayed ordinary differential equations). The time history before control is shown in Figs $12 \mathrm{a}$ and $\mathrm{b}$ at $\sigma_{1}=0.5(\Omega=100.5$, the highest amplitude point), while Fig. 12.c and d show the blade's actual frequency response. It is clear from the figure that the blade suffers from very high amplitudes before control mainly around $\Omega=100$. After control, the time history is shown in Fig. 13 at $\sigma_{1}=0$ with time delays inside and outside the safe region of time delays. From Fig. 13.a and b, we see that the output 

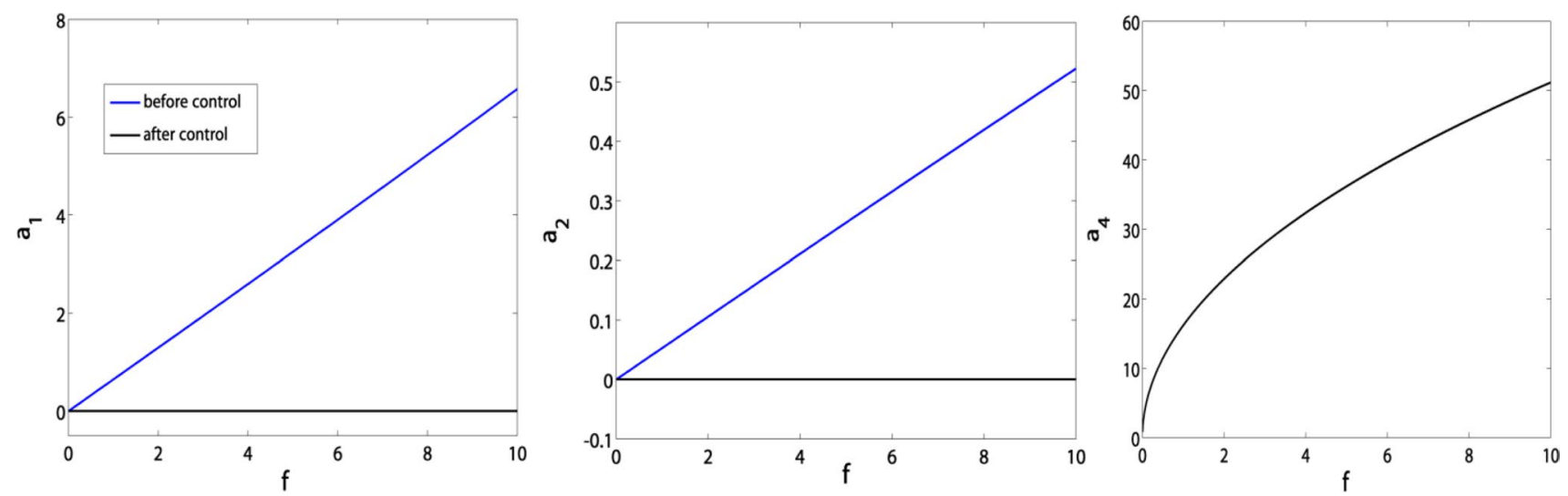

Fig. 10 Force response curves before control (blue) and after control (black) at $\sigma_{1}=+5, \tau_{1}+\tau_{2}=0.0005$
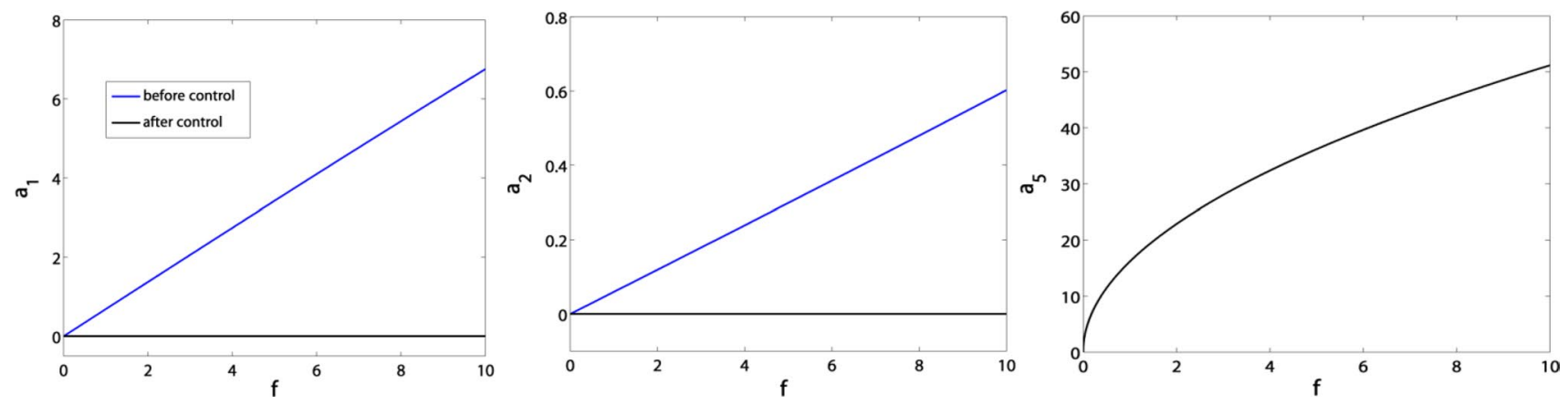

Fig. 11 Force response curves before control (blue) and after control (black) at $\sigma_{1}=-4.7, \tau_{1}+\tau_{2}=0.0005$
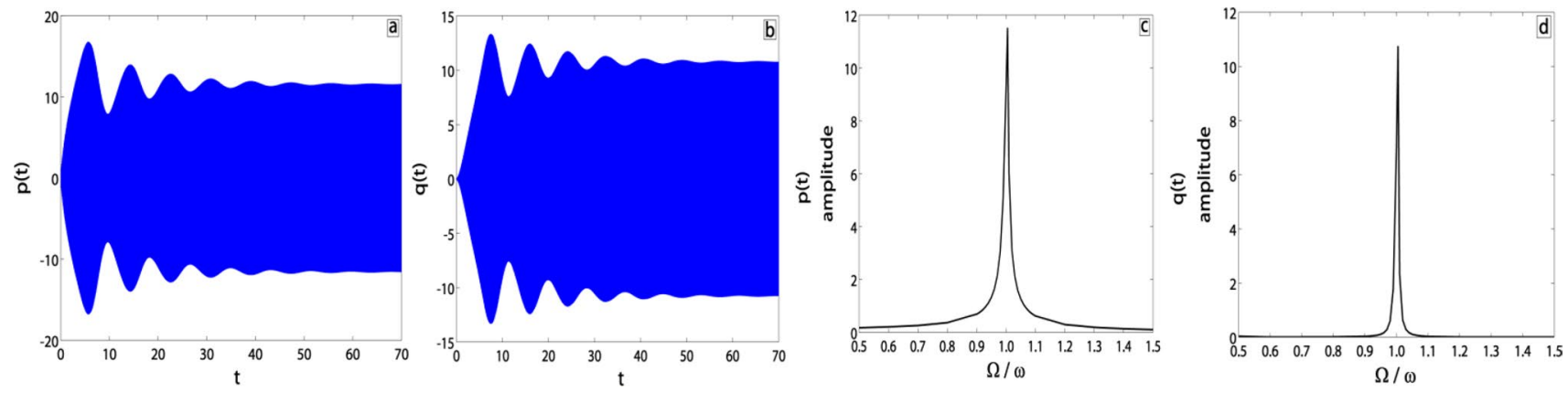

Fig. 12 Blade deflections before control: $\mathbf{a}$, $\mathbf{b}$ time history at $\Omega=100.5, \mathbf{c}$, $\mathbf{d}$ actual frequency response

steady amplitudes have been suppressed by about $99.85 \%$ for both blade deflections only if the time delays are still inside their safe region. Figures $13 \mathrm{~d}$ and e show the unstable motions of the blade and that is because of the time delays being out of their safe regions. Figures 14 and 15 show the time history after control at $\sigma_{1}=+5$ and -4.7 , respectively, with time delays inside and outside their safe regions. These figures assure the suppression of PPF high amplitude peaks at the aforementioned points in case of keeping the time delays inside the safe region, otherwise the system will exhibit unstable motions as shown.

Figure 16 shows a comparison of the actual frequency response of blade deflections before and after control. It is clear that the amplitudes are very high at $\Omega \cong 100$ (black curves). After applying the traditional PPF only, two peaks have appeared (red curves) on both sides of the uncontrolled peak. After applying the NSCs at each peak, the

\section{SN Applied Sciences}



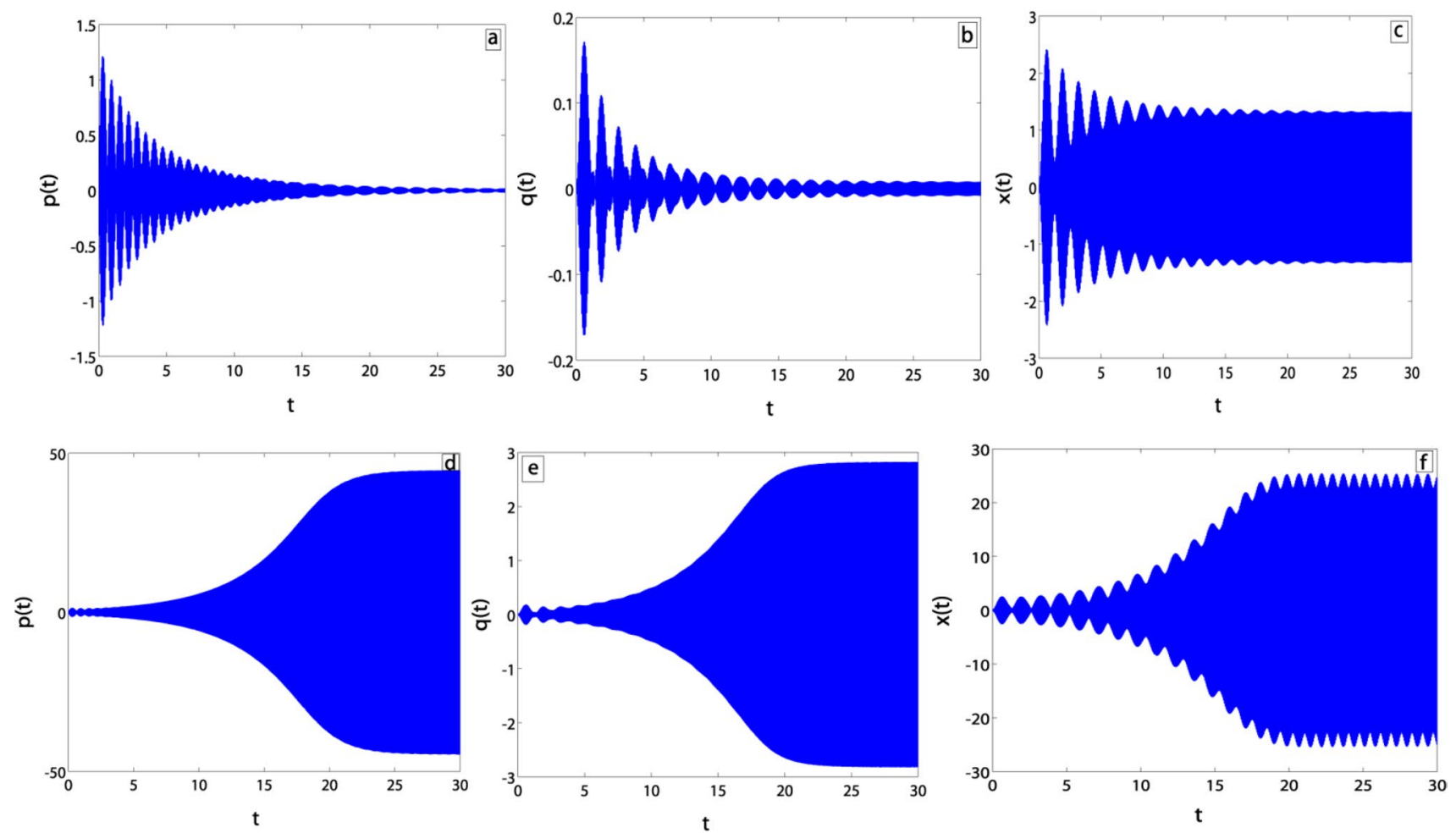

Fig. 13 Time history after control at $\sigma_{1}=0: \mathbf{a}, \mathbf{b}, \mathbf{c} \tau_{1}+\tau_{2}=0.0005, \mathbf{d}, \mathbf{e}, \mathbf{f} \tau_{1}+\tau_{2}=0.0011$
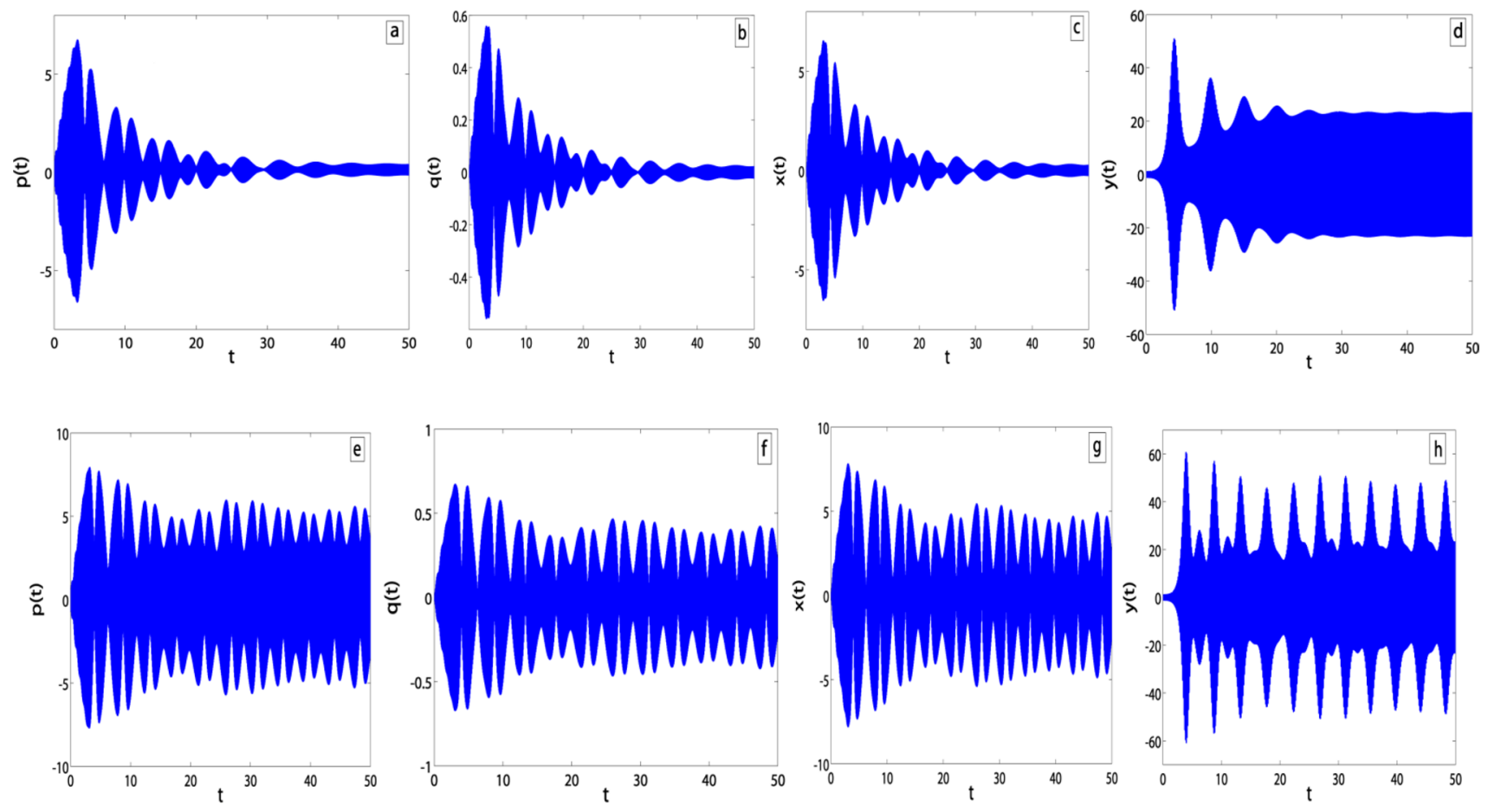

Fig. 14 Time history after control at $\sigma_{1}=+5: \mathbf{a}, \mathbf{b}, \mathbf{c}, \mathbf{d} \tau_{1}+\tau_{2}=0.0005, \mathbf{e}, \mathbf{f}, \mathbf{g}, \mathbf{h} \tau_{1}+\tau_{2}=0.0008$ 

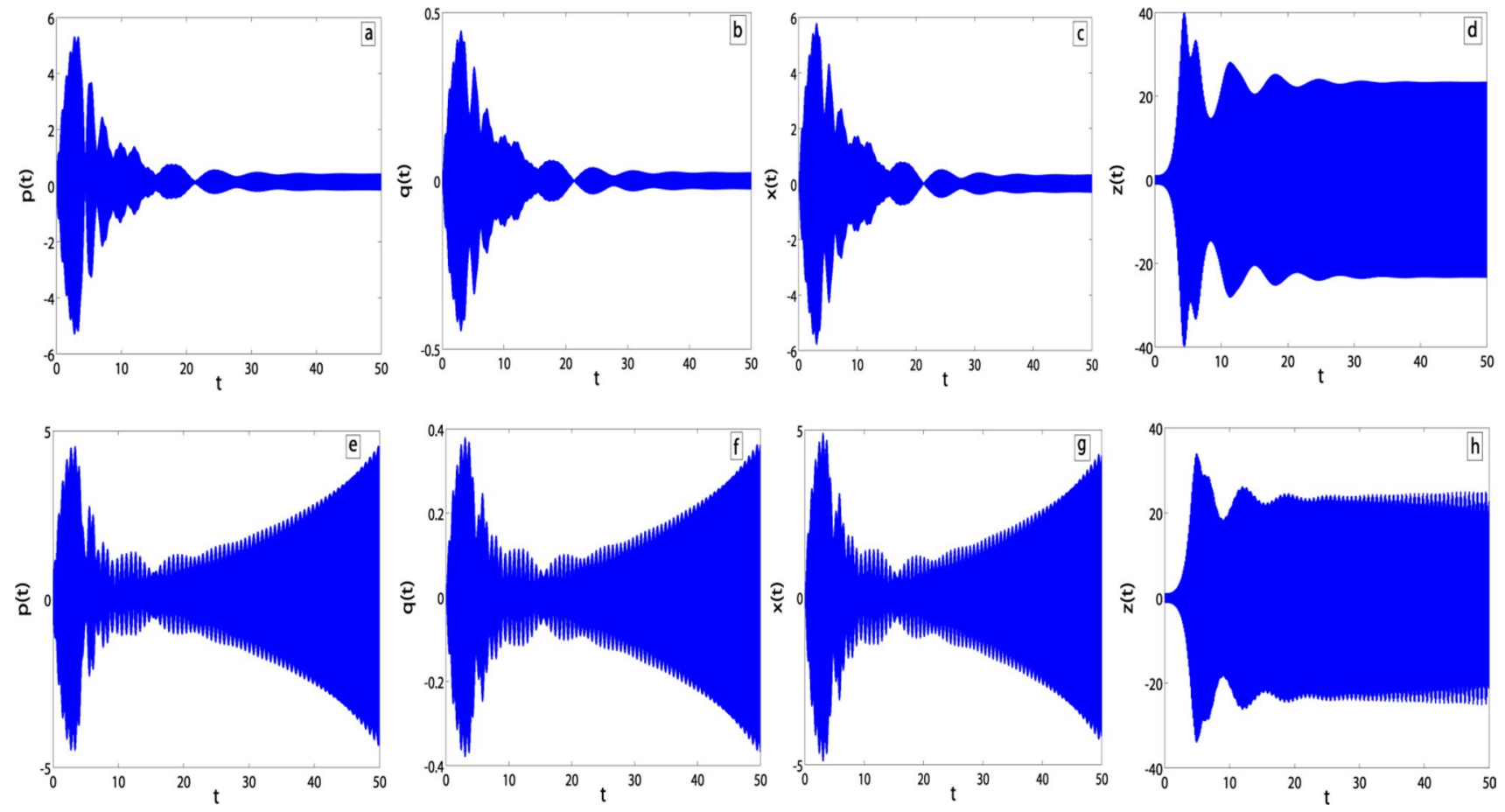

Fig. 15 Time history after control at $\sigma_{1}=-4.7: \mathbf{a}, \mathbf{b}, \mathbf{c}, \mathbf{d} \tau_{1}+\tau_{2}=0.0005, \mathbf{e}, \mathbf{f}, \mathbf{g}, \mathbf{h} \tau_{1}+\tau_{2}=0.0012$
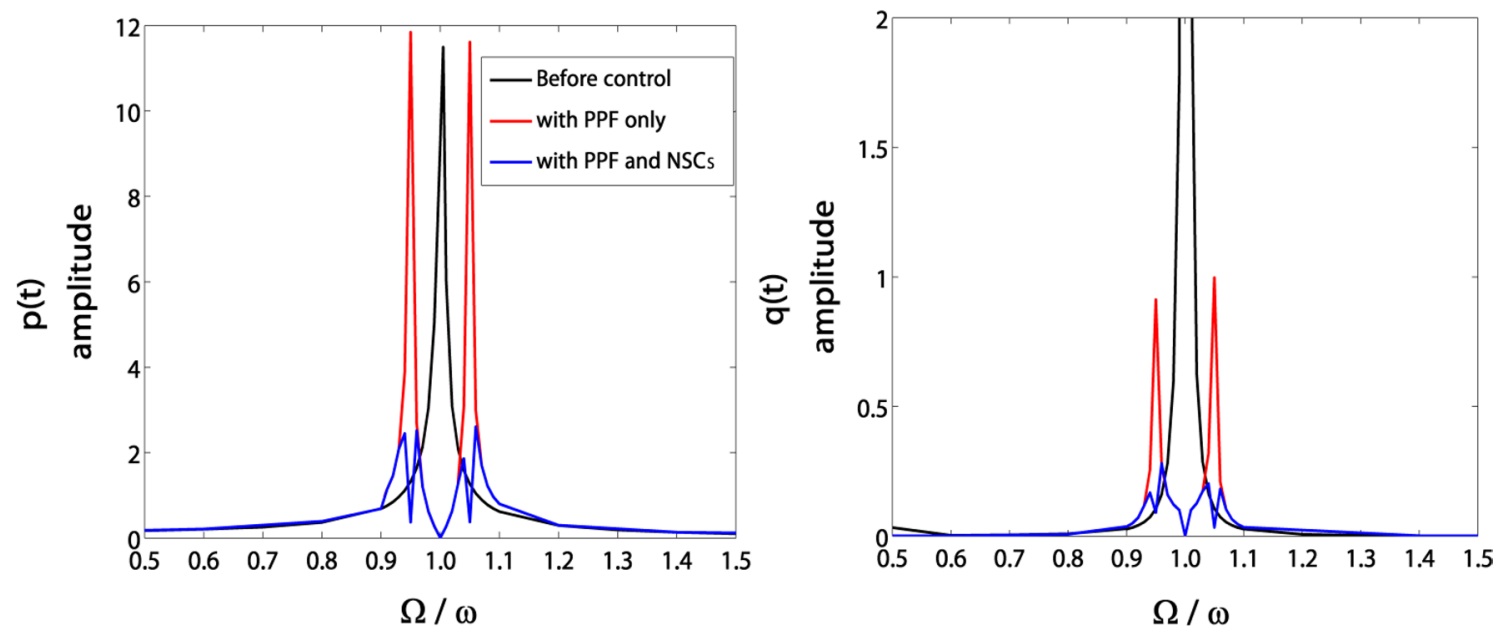

Fig. 16 Actual frequency response of blade deflections before and after control at $\tau_{1}+\tau_{2}=0.0005$

peaks are notched down via $V$-shapes (blue curves) to indicate the benefit of using the NSCs in this work.

\subsection{Perturbation curves validation}

Figures 17, 18, 19, 20 and 21 are plotted to validate the curves discussed in Sect. 3. Using the fourth-order Runge-Kutta technique, we have integrated Eq. 2 numerically. The lines refer to the approximate perturbation solutions while the circles and dots denote the ones resulted from the numerical integration. The figures show the degree of closeness between the numerical integration solutions and the approximate solutions before and after control. 

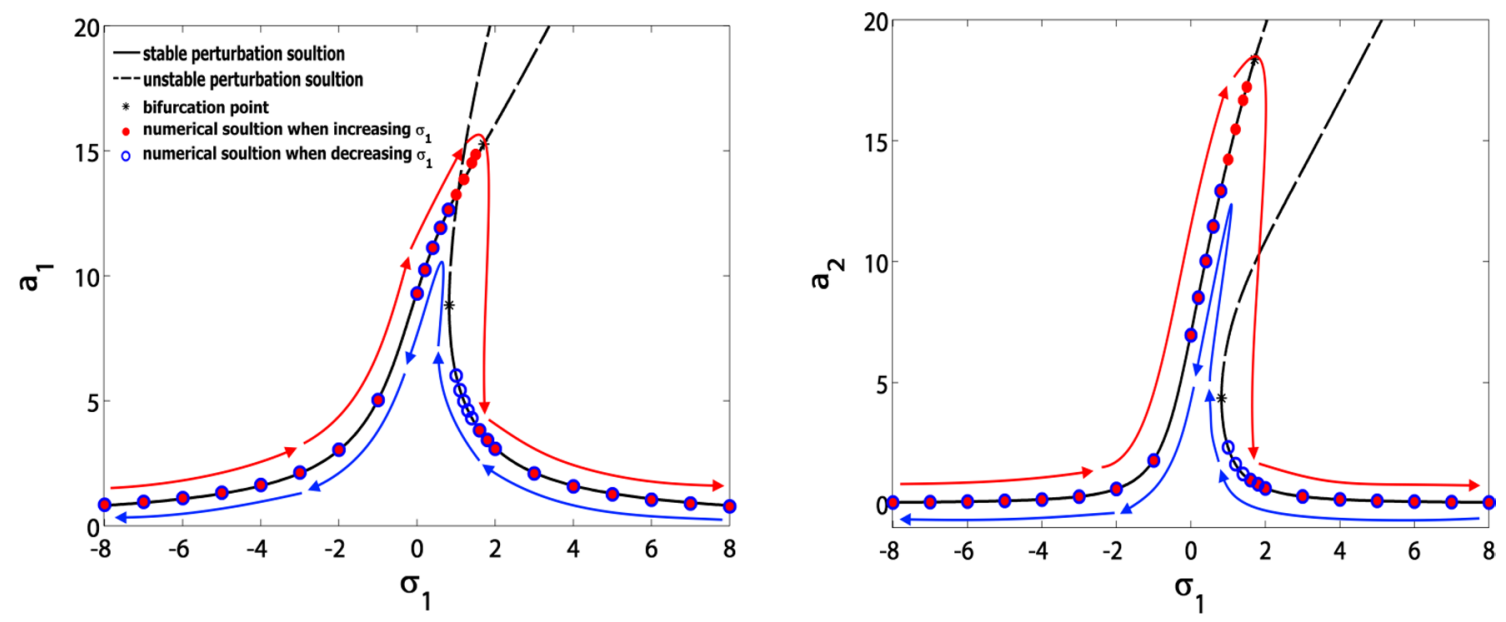

Fig. 17 Verification of frequency response curves (before control)
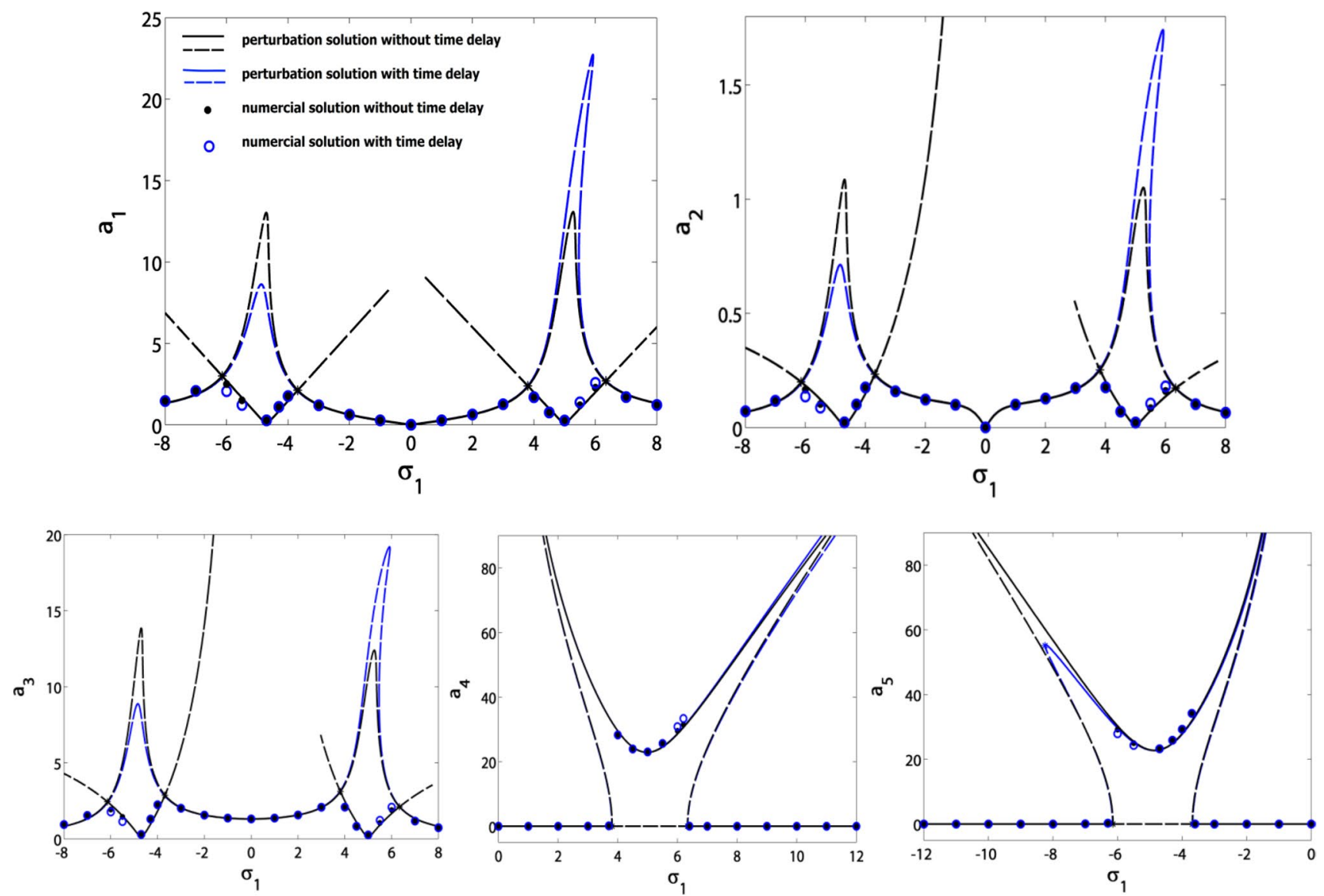

Fig. 18 Verification of frequency response curves after control without and with time delay

\section{Conclusions}

In this paper, a time delayed PPF has been coupled to a thin-walled pre-twisted blade. We have improved the performance of the traditional PPF controller by suppressing the two peaks to acceptable levels where that could be done by coupling dual NSCs to impose a V-curve at each one of the peaks. We have also checked the safe regions of time delays to keep a normal system operation. A 

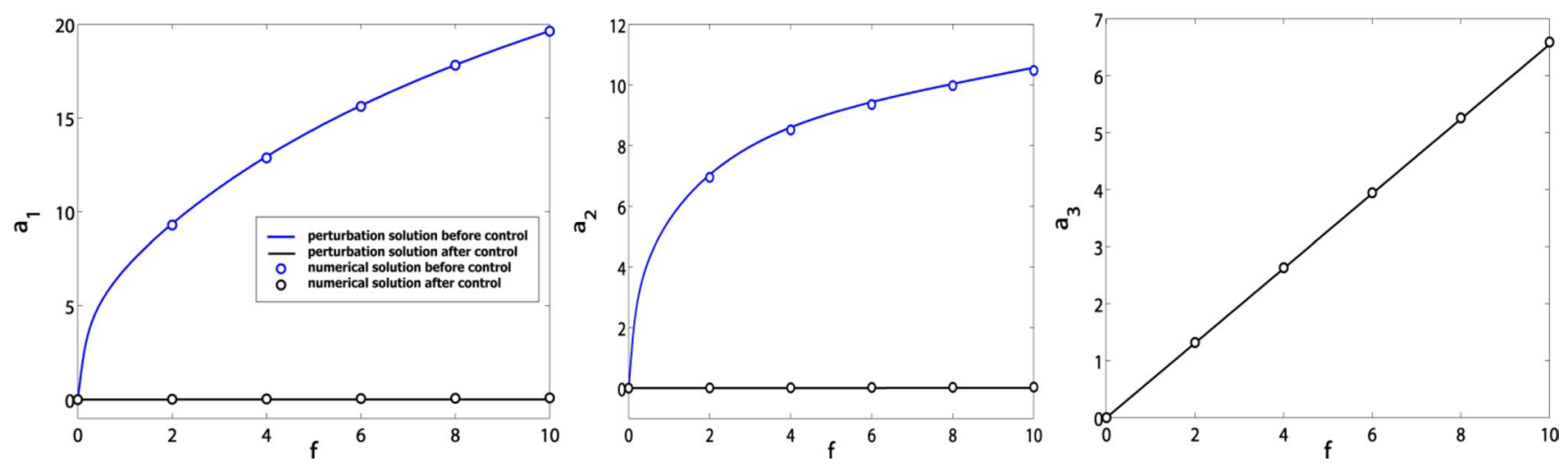

Fig. 19 Verification of force response curves at $\sigma_{1}=0$ and $\tau_{1}+\tau_{2}=0.005$ before and after control
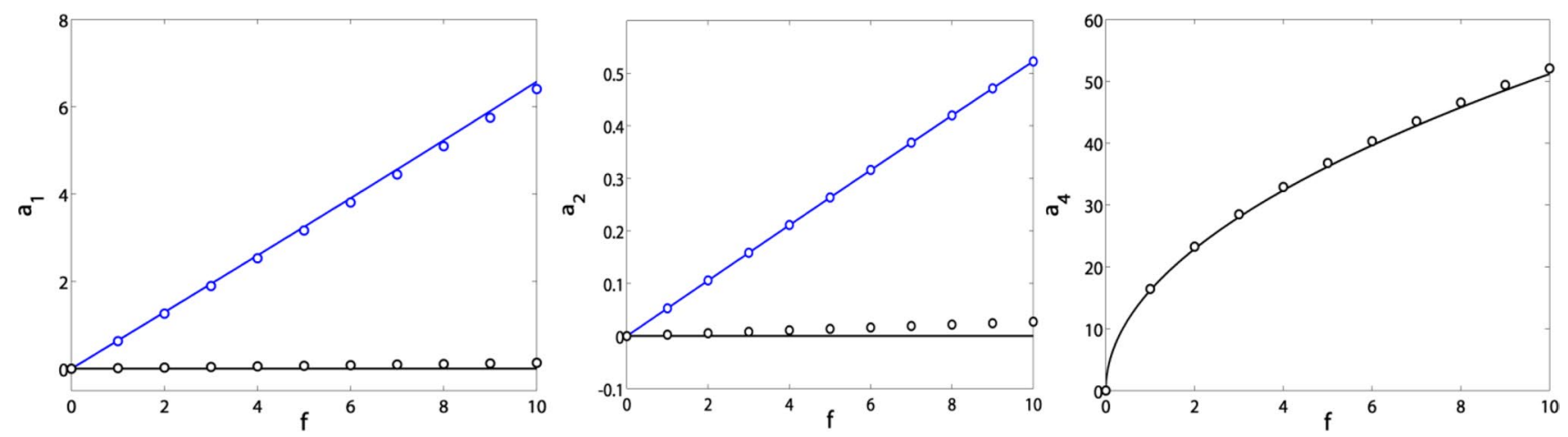

Fig. 20 Verification of force response curves at $\sigma_{1}=5$ and $\tau_{1}+\tau_{2}=0.005$ before and after control
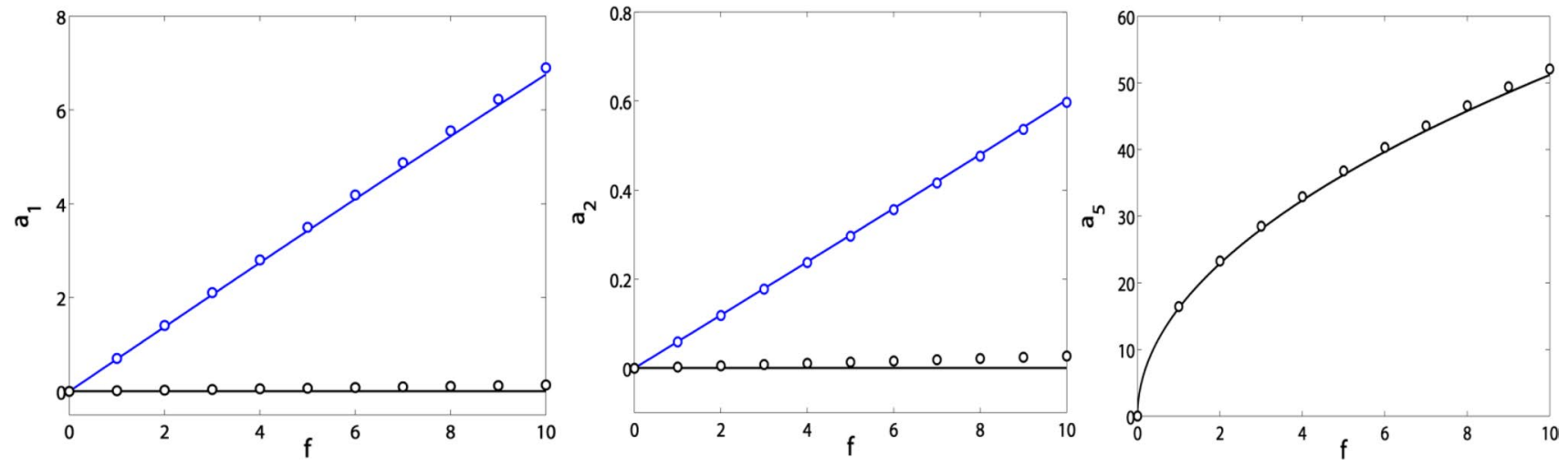

Fig. 21 Verification of force response curves at $\sigma_{1}=-4.7$ and $\tau_{1}+\tau_{2}=0.005$ before and after control

theoretical analysis has been conducted via multiple time scales method to derive approximate analytical solutions. The first method of Lyapunov was utilized to determine stable and unstable equilibrium solutions. Eventually, time history and validation curves have been plotted to verify the control algorithm. The overall study revealed the following conclusions. Before control, the blade crosssection suffered from jump phenomena, saddle-node and
Hopf bifurcations. But they were all eliminated after control. Both of blade deflection amplitudes have been suppressed by about $99.85 \%$ at $\sigma_{1}=0$ because of the PPF controller. Two peaks appeared at $\sigma_{1} \cong\{-4.7,+5\}$, but they were notched down via applying an NSC at each one of the peaks. The time delays $\tau_{1}$ and $\tau_{2}$ should be kept within the range $\tau_{1}+\tau_{2}<0.0007$ to guarantee a stable operation for a detuning range $-4.7 \leq \sigma_{1} \leq+5$. At safe time delays, 
the PPF peaks began to rise up higher but it did not matter as the V-curves were notching them down to minimum amplitudes. Increasing the excitation force amplitude $f$ before control increased the blade vibration amplitudes drastically which is a defect. After control, the amplitudes became almost constant regardless of increasing the excitation force amplitude $f$.

In the previous work regarding vibration control of the rotating blade, Younesian and Esmailzadeh [6] reduced the vibrations of the rotating beam using a time-increasing internal tensile force. They showed that the suppression system could effectively reduce vibration by about $50 \%$. The authors [19] applied a time delayed positive position feedback to the rotating blade model. They showed that the system amplitudes have been suppressed efficiently with a safe range of time delays $\tau_{1}+\tau_{2}<0.001$. In this paper, a combination of PPF and NSC controllers was applied to a rotating blade system subjected to primary excitation via MFC actuators. Addition of the NSCs has improved the PPF operation such that the two peaks created by the Classical PPF have been notched down through $V$-shaped curves. The time delays $\tau_{1}$ and $\tau_{2}$ should be within the range $\tau_{1}+\tau_{2}<0.0007$ to guarantee a stable operation for a detuning range $-4.7 \leq \sigma_{1} \leq+5$. Increasing the excitation force (after control) produced almost small constant output amplitudes unlike the case before control. Upon the mentioned parameters in this paper, the control method has reduced the vibrations by about $99.85 \%$ at $\sigma_{1}=0$.

Funding This research received no specific grant from any funding agency in the public, commercial, or not-for-profit sectors.

\section{Compliance with ethical standards}

Conflict of interest The author declares that he has no conflict of interest in preparing this article.

\section{Appendix A}

\section{See Fig. 22}

Consider a thin-walled cantilever blade of length $L$ and thickness $h$ attached to a rigid hub of radius $R_{0}$. This rigid hub rotates about its axis with a speed $\Omega$. During rotation and due to the blade's mass, it is assumed that the blade can be excited by a periodic force $f \cos \Omega$ t carried on a steady force $f_{0}$ to make the overall excitation as $F=f_{0}+f \cos \Omega$ t. An angle $\gamma$ is created thanks to allowing the blade to vibrate flexurally in the rotation as shown in Fig. 22. For deriving the analytical model, we make the following assumptions:

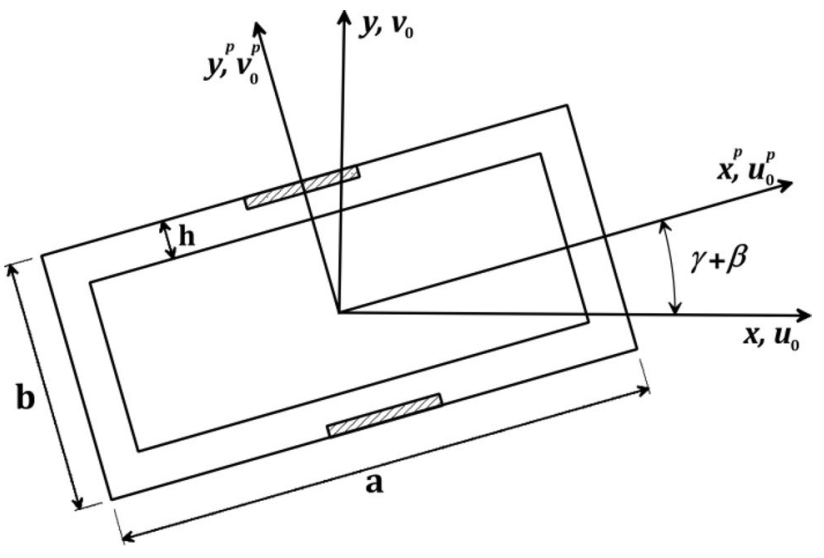

Fig. 22 Rotating blade's cross-section

(1) The blade's cross-section is still un-deformed during operation.

(2) The ratio $h / r$ can be neglected compared to unity where $r$ is the blade's radius of curvature.

(3) The shear force in the transverse direction can be neglected.

(4) The axial elongation $w_{0}$ can be neglected compared to the deflections $u_{0}$ and $v_{0}$.

The rotary coordinate axes $x^{p}$ and $y^{p}$ can be related to the fixed coordinate axes $x$ and $y$ by the following relations:

$x=x^{p} \cos (\gamma+\beta)-y^{p} \sin (\gamma+\beta)$

$y=x^{p} \sin (\gamma+\beta)+y^{p} \cos (\gamma+\beta)$

where $\beta=\beta_{0} z / L$ is the twist angle of the whole blade along the $z$ axis, and $\beta_{0}$ is the twist angle at the blades' tip. An extended Hamilton principle can be utilized to derive the blade's equations of motion. It is formulated as.

$\int_{0}^{t}(\delta K-\delta U+\delta W) d t=0$

Where $K$ and $U$ are the kinetic and strain energy, respectively, $W$ is the virtual work produced by external forces, $t$ is the time, and $\delta$ is the variation operator. In $[4,5]$, the authors have derived in detail and extracted the dimensionless equations governing the blades nonlinear vibrations as:

$$
\begin{aligned}
\ddot{u}_{0} & -F^{2} u_{0}-F^{2}\left[R(z) u_{0}^{\prime \prime}+R^{\prime}(z) u_{0}^{\prime}\right]+\alpha \Delta T u_{0}^{\prime \prime}-\left[\alpha_{3}(z) v_{0}^{\prime \prime}-\alpha_{6}(z) u_{0}^{\prime \prime}\right]^{\prime \prime} \\
= & u_{0}^{\prime}\left(u_{0}^{\prime} u_{0}^{\prime \prime}+v_{0}^{\prime} v_{0}^{\prime \prime}\right)+u_{0}^{\prime \prime}\left[\frac{1}{2}\left(u_{0}^{\prime}\right)^{2}+\frac{1}{2}\left(v_{0}^{\prime}\right)^{2}\right]-\dot{F}\left(R_{0}+z\right)+p_{x}
\end{aligned}
$$




$$
\begin{aligned}
\ddot{v}_{0} & -F^{2}\left[R(z) v_{0}^{\prime \prime}+R^{\prime}(z) v_{0}^{\prime}\right]+\alpha \Delta T v_{0}^{\prime \prime}-\left[\alpha_{3}(z) u_{0}^{\prime \prime}-\alpha_{4}(z) v_{0}^{\prime \prime}\right]^{\prime \prime} \\
& =v_{0}^{\prime}\left(u_{0}^{\prime} u_{0}^{\prime \prime}+v_{0}^{\prime} v_{0}^{\prime \prime}\right)+v_{0}^{\prime \prime}\left[\frac{1}{2}\left(u_{0}^{\prime}\right)^{2}+\frac{1}{2}\left(v_{0}^{\prime}\right)^{2}\right]+p_{y}
\end{aligned}
$$

where the dots and primes refer to partial differentiation w.r.t $t$ and $z$, respectively. A discretization is done to Eq. A3 applying the one-term Galerkin technique by expressing the blade's modes in the form:

$u_{0}=G(z) p(t)$

$v_{0}=G(z) q(t)$

where $p(t)$ and $q(t)$ are the temporal deflections of the studied blade, $G(z)$ is the linear free undamped mode of the studied blade which has the following form:

$G(z)=\cosh (\Gamma z)-\cos (\Gamma z)-\left[\frac{\cosh \Gamma+\cos \Gamma}{\sinh \Gamma+\sin \Gamma}\right][\sinh (\Gamma z)-\sin (\Gamma z)]$

where $\Gamma$ is the solution of the relation $\cosh \Gamma \cos \Gamma+1=0$. Substituting Eq. A4 and A5 into Eq. A3, multiplying Eq. A3 by $G(z)$, then integrating the results w.r.t $z$ from 0 to 1 yields a 2DOF nonlinear system of ODEs as:

$$
\begin{aligned}
& \alpha_{5} \ddot{p}+\alpha_{11} \dot{p}+\left(\alpha_{13}+\alpha_{17}-\alpha_{10} f_{0}^{2}\right) p+\alpha_{2} \dot{q}+\left(\alpha_{14}+\alpha_{8}\right) q \\
& -2 \alpha_{10} p f_{0} f \cos (\Omega t)-\alpha_{10} p f^{2} \cos ^{2}(\Omega t)+\alpha_{6} p^{3}+\alpha_{6} p q^{2} \\
& =\alpha_{9} \Omega f \sin (\Omega t)
\end{aligned}
$$

$\alpha_{5} \ddot{q}+\alpha_{21} \dot{q}+\left(\alpha_{24}+\alpha_{27}-\alpha_{20} f_{0}^{2}\right) q+\alpha_{2} \dot{p}+\left(\alpha_{23}+\alpha_{8}\right) p$

$-2 \alpha_{20} q f_{0} f \cos (\Omega t)-\alpha_{20} q f^{2} \cos ^{2}(\Omega t)+\alpha_{6} q^{3}+\alpha_{6} p^{2} q=0$

For a strong coupling between the two modes, we will consider $\omega_{1} \cong \omega_{2}=\omega$ as mentioned in Ref. [4], then dividing by $\alpha_{5}$ yields:

$$
\begin{aligned}
& \ddot{p}+2 \mu \dot{p}+\omega^{2} p+\beta_{13} \dot{q}+\beta_{11} q+\beta_{5} p q^{2}+\beta_{5} p^{3} \\
& =2 f_{0} f \beta_{14} p \cos (\Omega t)+f^{2} \beta_{14} p \cos ^{2}(\Omega t)+f \beta_{16} \Omega \sin (\Omega t)
\end{aligned}
$$

$$
\begin{gathered}
\ddot{q}+2 \mu \dot{q}+\omega^{2} q+\beta_{22} \dot{p}+\beta_{21} p+\beta_{5} p^{2} q+\beta_{5} q^{3} \\
=2 f_{0} f \beta_{24} q \cos (\Omega t)+f^{2} \beta_{24} q \cos ^{2}(\Omega t)
\end{gathered}
$$

where the parameters of Eqs. $A 3, A 6$, and $A 7$ are given in $[4,5]$.

\section{Appendix B}

Substituting Eq. $3-5$ into Eq. 2 yields.

$$
\begin{aligned}
{\left[D_{0}^{2}+\right.} & \left.2 \varepsilon D_{1} D_{0}\right]\left(p_{0}+\varepsilon p_{1}\right)+2 \varepsilon \hat{\mu} D_{0} p_{0}+\omega^{2}\left(p_{0}+\varepsilon p_{1}\right) \\
+ & \varepsilon \hat{\beta}_{13} D_{0} q_{0}+\varepsilon \hat{\beta}_{11} q_{0}+\varepsilon \hat{\beta}_{5} p_{0} q_{0}^{2}+\varepsilon \hat{\beta}_{5} p_{0}^{3} \\
= & 2 \varepsilon f_{0} f \hat{\beta}_{14} p_{0} \cos (\Omega t)+\varepsilon f^{2} \hat{\beta}_{14} p_{0} \cos ^{2}(\Omega t) \\
& +\varepsilon f \hat{\beta}_{16} \Omega \sin (\Omega t)++\varepsilon \hat{c}_{1} x_{0 \tau_{2}}+\varepsilon \hat{c}_{2}\left(y_{0 \tau_{2}}^{2}+z_{0 \tau_{2}}^{2}\right)+O\left(\varepsilon^{2}\right)
\end{aligned}
$$

$$
\begin{aligned}
& {\left[D_{0}^{2}+2 \varepsilon D_{1} D_{0}\right]\left(q_{0}+\varepsilon q_{1}\right)+2 \varepsilon \hat{\mu} D_{0} q_{0}+\omega^{2}\left(q_{0}+\varepsilon q_{1}\right)} \\
& \quad+\varepsilon \hat{\beta}_{22} D_{0} p_{0}+\varepsilon \hat{\beta}_{21} p_{0}+\varepsilon \hat{\beta}_{5} p_{0}^{2} q_{0}+\varepsilon \hat{\beta}_{5} q_{0}^{3} \\
& \quad=2 \varepsilon f_{0} f \hat{\beta}_{24} q_{0} \cos (\Omega t)+\varepsilon f^{2} \hat{\beta}_{24} q_{0} \cos ^{2}(\Omega t)+O\left(\varepsilon^{2}\right)
\end{aligned}
$$

$$
\begin{aligned}
& {\left[D_{0}^{2}+2 \varepsilon D_{1} D_{0}\right]\left(x_{0}+\varepsilon x_{1}\right)+2 \varepsilon \hat{\mu}_{1} D_{0} x_{0}+\omega_{1}^{2}\left(x_{0}+\varepsilon x_{1}\right)} \\
& \quad=\varepsilon \hat{C}_{3} p_{0 \tau_{1}}+O\left(\varepsilon^{2}\right)
\end{aligned}
$$

$$
\begin{aligned}
& {\left[D_{0}^{2}+2 \varepsilon D_{1} D_{0}\right]\left(y_{0}+\varepsilon y_{1}\right)+2 \varepsilon \hat{\mu}_{2} D_{0} y_{0}+\omega_{2}^{2}\left(y_{0}+\varepsilon y_{1}\right)} \\
& \quad=\varepsilon \hat{c}_{4} p_{0 \tau_{1}} y_{0}+O\left(\varepsilon^{2}\right)
\end{aligned}
$$

$$
\begin{aligned}
& {\left[D_{0}^{2}+2 \varepsilon D_{1} D_{0}\right]\left(y_{0}+\varepsilon y_{1}\right)+2 \varepsilon \hat{\mu}_{2} D_{0} y_{0}+\omega_{2}^{2}\left(y_{0}+\varepsilon y_{1}\right)} \\
& \quad=\varepsilon \hat{c}_{4} p_{0 \tau_{1}} y_{0}+O\left(\varepsilon^{2}\right)
\end{aligned}
$$

Comparing the coefficients of equal powers of $\varepsilon$ on both sides of Eq. B1 yields

$O\left(\varepsilon^{0}\right):$

$D_{0}^{2} p_{0}+\omega^{2} p_{0}=0$

$D_{0}^{2} q_{0}+\omega^{2} q_{0}=0$

$D_{0}^{2} x_{0}+\omega_{1}^{2} x_{0}=0$

$D_{0}^{2} y_{0}+\omega_{2}^{2} y_{0}=0$

$D_{0}^{2} z_{0}+\omega_{3}^{2} z_{0}=0$

$O\left(\varepsilon^{1}\right):$

$$
\begin{aligned}
D_{0}^{2} p_{1}+\omega^{2} p_{1}= & -2 D_{1} D_{0} p_{0}-2 \hat{\mu} D_{0} p_{0}-\hat{\beta}_{13} D_{0} q_{0}-\hat{\beta}_{11} q_{0} \\
& -\hat{\beta}_{5} p_{0} q_{0}^{2}-\hat{\beta}_{5} p_{0}^{3}+f_{0} f \hat{\beta}_{14} p_{0}\left(e^{i \Omega T_{0}}+e^{-i \Omega T_{0}}\right) \\
& +\frac{f^{2} \hat{\beta}_{14}}{4} p_{0}\left(e^{i \Omega T_{0}}+e^{-i \Omega T_{0}}\right)^{2}-i \frac{f \hat{\beta}_{16} \Omega}{2}\left(e^{i \Omega T_{0}}-e^{-i \Omega T_{0}}\right) \\
& +\hat{c}_{1} x_{0 \tau_{2}}+\hat{c}_{2}\left(y_{0 \tau_{2}}^{2}+z_{0 \tau_{2}}^{2}\right)
\end{aligned}
$$




$$
\begin{aligned}
D_{0}^{2} q_{1}+\omega^{2} q_{1}= & -2 D_{1} D_{0} q_{0}-2 \hat{\mu} D_{0} q_{0}-\hat{\beta}_{22} D_{0} p_{0}-\hat{\beta}_{21} p_{0} \\
& -\hat{\beta}_{5} p_{0}^{2} q_{0}-\hat{\beta}_{5} q_{0}^{3}+f_{0} f \hat{\beta}_{24} q_{0}\left(e^{i \Omega T_{0}}+e^{-i \Omega T_{0}}\right) \\
& +\frac{f^{2} \hat{\beta}_{24}}{4} q_{0}\left(e^{i \Omega T_{0}}+e^{-i \Omega T_{0}}\right)^{2}
\end{aligned}
$$

$D_{0}^{2} x_{1}+\omega_{1}^{2} x_{1}=-2 D_{1} D_{0} x_{0}-2 \hat{\mu}_{1} D_{0} x_{0}+\hat{c}_{3} p_{0 \tau_{1}}$

$D_{0}^{2} y_{1}+\omega_{2}^{2} y_{1}=-2 D_{1} D_{0} y_{0}-2 \hat{\mu}_{2} D_{0} y_{0}+\hat{c}_{4} p_{0 \tau_{1}} y_{0}$

$D_{0}^{2} z_{1}+\omega_{3}^{2} z_{1}=-2 D_{1} D_{0} z_{0}-2 \hat{\mu}_{3} D_{0} z_{0}+\hat{c}_{4} p_{0 \tau_{1}} z_{0}$

The solutions of Eq. B3 can be expressed as.

$$
\begin{aligned}
& p_{0}=A_{1} e^{i \omega T_{0}}+\bar{A}_{1} e^{-i \omega T_{0}} \\
& q_{0}=A_{2} e^{i \omega T_{0}}+\bar{A}_{2} e^{-i \omega T_{0}} \\
& x_{0}=A_{3} e^{i \omega_{1} T_{0}}+\bar{A}_{3} e^{-i \omega_{1} T_{0}} \\
& y_{0}=A_{4} e^{i \omega_{2} T_{0}}+\bar{A}_{4} e^{-i \omega_{2} T_{0}} \\
& z_{0}=A_{5} e^{i \omega_{3} T_{0}}+\bar{A}_{5} e^{-i \omega_{3} T_{0}}
\end{aligned}
$$

while the delayed signals $p_{0 \tau_{1},}, x_{0 \tau_{2},}, y_{0 \tau_{2}}$ and $z_{0 \tau_{2}}$ can be expressed using Taylor sers as.

$$
\begin{aligned}
& p_{0 \tau_{1}} \cong A_{1}\left(T_{1}\right) e^{i \omega\left(T_{0}-\tau_{1}\right)}+\bar{A}_{1}\left(T_{1}\right) e^{-i \omega\left(T_{0}-\tau_{1}\right)} \\
& x_{0 \tau_{2}} \cong A_{3}\left(T_{1}\right) e^{i \omega_{1}\left(T_{0}-\tau_{2}\right)}+\bar{A}_{3}\left(T_{1}\right) e^{-i \omega_{1}\left(T_{0}-\tau_{2}\right)} \\
& y_{0 \tau_{2}} \cong A_{4}\left(T_{1}\right) e^{i \omega_{2}\left(T_{0}-\tau_{2}\right)}+\bar{A}_{4}\left(T_{1}\right) e^{-i \omega_{2}\left(T_{0}-\tau_{2}\right)} \\
& z_{0 \tau_{2}} \cong A_{5}\left(T_{1}\right) e^{i \omega_{3}\left(T_{0}-\tau_{2}\right)}+\bar{A}_{5}\left(T_{1}\right) e^{-i \omega_{3}\left(T_{0}-\tau_{2}\right)}
\end{aligned}
$$

Substituting Eq. 4 and 5 into Eq. B3 yields.

$$
\begin{aligned}
D_{0}^{2} p_{1}+\omega^{2} p_{1}= & \left(-2 i \omega D_{1} A_{1}-2 i \hat{\mu} \omega A_{1}-i \hat{\beta}_{13} \omega A_{2}-\hat{\beta}_{11} A_{2}\right. \\
& \left.-2 \hat{\beta}_{5} A_{1} A_{2} \bar{A}_{2}-\hat{\beta}_{5} \bar{A}_{1} A_{2}^{2}-3 \hat{\beta}_{5} A_{1}^{2} \bar{A}_{1}+\frac{f^{2} \beta_{14}}{2} A_{1}\right) e^{i \omega T_{0}} \\
& +f_{0} f \hat{\beta}_{14} \bar{A}_{1} e^{i T_{0}(\Omega-\omega)} \frac{f^{2} \hat{\beta}_{14}}{4} \bar{A}_{1} e^{i T_{0}(2 \Omega-\omega)}-i \frac{f \hat{\beta}_{16} \Omega}{2} e^{i \Omega T_{0}} \\
& +\hat{c}_{1} A_{3} e^{i \omega_{1}\left(T_{0}-\tau_{2}\right)}+\hat{c}_{2}\left(A_{4}^{2} e^{2 i \omega_{2}\left(T_{0}-\tau_{2}\right)}+A_{5}^{2} e^{2 i \omega_{3}\left(T_{0}-\tau_{2}\right)}\right) \\
& +N S T+c c
\end{aligned}
$$

$$
\begin{aligned}
D_{0}^{2} q_{1}+\omega^{2} q_{1}=( & -2 i \omega D_{1} A_{2}-2 i \hat{\mu} \omega A_{2}-i \hat{\beta}_{22} \omega A_{1}-\hat{\beta}_{21} A_{1} \\
& \left.-2 \hat{\beta}_{5} A_{1} \bar{A}_{1} A_{2}-\hat{\beta}_{5} A_{1}^{2} \bar{A}_{2}-3 \hat{\beta}_{5} A_{2}^{2} \bar{A}_{2}+\frac{f^{2} \hat{\beta}_{24}}{2} A_{2}\right) e^{i \omega T_{0}} \\
& +f_{0} f \hat{\beta}_{24} \bar{A}_{2} e^{i T_{0}(\Omega-\omega)}+\frac{f^{2} \hat{\beta}_{24}}{4} \bar{A}_{2} e^{i T_{0}(2 \Omega-\omega)}+N S T+c c
\end{aligned}
$$

$$
\begin{aligned}
D_{0}^{2} x_{1}+\omega_{1}^{2} x_{1}= & \left(-2 i \omega_{1} D_{1} A_{3}-2 i \hat{\mu}_{1} \omega_{1} A_{3}\right) e^{i \omega_{1} T_{0}} \\
& +\hat{c}_{3} A_{1} e^{i \omega\left(T_{0}-\tau_{1}\right)}+N S T+c C
\end{aligned}
$$

$$
\begin{aligned}
D_{0}^{2} y_{1}+\omega_{2}^{2} y_{1}= & \left(-2 i \omega_{2} D_{1} A_{4}-2 i \hat{\mu}_{2} \omega_{2} A_{4}\right) e^{i \omega_{2} T_{0}} \\
& +\hat{c}_{4} A_{1} \bar{A}_{4} e^{-i \omega \tau_{1}} e^{i T_{0}\left(\omega-\omega_{2}\right)}+N S T+c C
\end{aligned}
$$

$$
\begin{aligned}
D_{0}^{2} z_{1}+\omega_{3}^{2} z_{1}= & \left(-2 i \omega_{3} D_{1} A_{5}-2 i \hat{\mu}_{3} \omega_{3} A_{5}\right) e^{i \omega_{3} T_{0}} \\
& +\hat{c}_{4} A_{1} \bar{A}_{5} e^{-i \omega \tau_{1}} e^{i T_{0}\left(\omega-\omega_{3}\right)}+N S T+c C
\end{aligned}
$$

where NST refers to the non-secular terms while $c c$ refers to the complex conjugate of the preceding terms. The detuning parameters $\sigma_{1}, \sigma_{2}, \sigma_{3}$, and $\sigma_{4}$ are introduced such that.

$\Omega=\omega+\sigma_{1}$

$\omega_{1}=\omega+\sigma_{2}$

$2 \omega_{2}=\omega+\sigma_{3}$

$2 \omega_{3}=\omega+\sigma_{4}$

to be inserted into Eq. B6, then eliminating the secular terms and using Eq. 3 and 5.a to return every scaled quantity to its real value yields.

$$
\begin{aligned}
& -2 i \omega \dot{A}_{1}-2 i \mu \omega A_{1}-i \omega \beta_{13} A_{2}-\beta_{11} A_{2}-2 \beta_{5} A_{1} A_{2} \bar{A}_{2}-\beta_{5} \bar{A}_{1} A_{2}^{2} \\
& -3 \beta_{5} A_{1}^{2} \bar{A}_{1}+\frac{\beta_{14} f^{2}}{2} A_{1}+\frac{\beta_{14} f^{2}}{4} \bar{A}_{1} e^{2 i \sigma_{1} t}+c_{1} A_{3} e^{i\left(\sigma_{2} t-\omega_{1} \tau_{2}\right)} \\
& +c_{2}\left(A_{4}^{2} e^{i\left(\sigma_{3} t-2 \omega_{2} \tau_{2}\right)}+A_{5}^{2} e^{i\left(\sigma_{4} t-2 \omega_{3} \tau_{2}\right)}\right)-\frac{i}{2} \beta_{16} \Omega f e^{i \sigma_{1} t}=0 \\
& -2 i \omega \dot{A}_{2}-2 i \mu \omega A_{2}-i \omega \beta_{22} A_{1}-\beta_{21} A_{1}-2 \beta_{5} A_{1} \bar{A}_{1} A_{2}-\beta_{5} A_{1}^{2} \bar{A}_{2} \\
& -3 \beta_{5} A_{2}^{2} \bar{A}_{2}+\frac{\beta_{24} f^{2}}{2} A_{2}+\frac{\beta_{24} f^{2}}{4} \bar{A}_{2} e^{2 i \sigma_{1} t}=0 \\
& -2 i \omega_{1} \dot{A}_{3}-2 i \mu_{1} \omega_{1} A_{3}+c_{3} A_{1} e^{-i\left(\sigma_{2} t+\omega \tau_{1}\right)}=0 \\
& -2 i \omega_{2} \dot{A}_{4}-2 i \mu_{2} \omega_{2} A_{4}+c_{4} A_{1} \bar{A}_{4} e^{-i\left(\sigma_{3} t+\omega \tau_{1}\right)}=0
\end{aligned}
$$

$$
-2 i \omega_{3} \dot{A}_{5}-2 i \mu_{3} \omega_{3} A_{5}+c_{4} A_{1} \bar{A}_{5} e^{-i\left(\sigma_{4} t+\omega \tau_{1}\right)}=0
$$


The quantities $A_{n}(n=1, \ldots, 5)$ can be rewritten in polar form such that.

$A_{n}=\frac{a_{n}}{2} e^{i \delta_{n}} \Rightarrow \dot{A}_{n}=\frac{\dot{a}_{n}}{2} e^{i \delta_{n}}+i \frac{a_{n}}{2} \dot{\delta}_{n} e^{i \delta_{n}}$

where $a_{n}$ and $\delta_{n}$ are temporal functions. Substituting Eq. B9 into Eq. B8 and dividing by $e^{i \delta_{n}}$, then simplifying give us.

$$
\begin{aligned}
& -i \omega \dot{a}_{1}+\omega a_{1} \dot{\delta}_{1}-i \mu \omega a_{1}-i \frac{\omega \beta_{13}}{2} a_{2}\left(\cos \phi_{2}+i \sin \phi_{2}\right) \\
& -\frac{\beta_{11}}{2} a_{2}\left(\cos \phi_{2}+i \sin \phi_{2}\right)-\frac{\beta_{5}}{4} a_{1} a_{2}^{2} \\
& -\frac{\beta_{5}}{8} a_{1} a_{2}^{2}\left(\cos \left(2 \phi_{2}\right)+i \sin \left(2 \phi_{2}\right)\right)-\frac{3 \beta_{5}}{8} a_{1}^{3} \\
& +\frac{\beta_{14} f^{2}}{8} a_{1}\left(\cos \left(2 \phi_{1}\right)+i \sin \left(2 \phi_{1}\right)\right) \\
& +\frac{\beta_{14} f^{2}}{4} a_{1}-i \frac{\beta_{16} \Omega f}{2}\left(\cos \phi_{1}+i \sin \phi_{1}\right) \\
& +\frac{c_{1}}{2} a_{3}\left(\cos \left(\phi_{3}-\omega_{1} \tau_{2}\right)+i \sin \left(\phi_{3}-\omega_{1} \tau_{2}\right)\right) \\
& +\frac{c_{2}}{4} a_{4}^{2}\left(\cos \left(\phi_{4}-2 \omega_{2} \tau_{2}\right)+i \sin \left(\phi_{4}-2 \omega_{2} \tau_{2}\right)\right) \\
& +\frac{c_{2}}{4} a_{5}^{2}\left(\cos \left(\phi_{5}-2 \omega_{3} \tau_{2}\right)+i \sin \left(\phi_{5}-2 \omega_{3} \tau_{2}\right)\right)=0
\end{aligned}
$$

$$
\begin{aligned}
& -i \omega \dot{a}_{2}+\omega a_{2} \dot{\delta}_{2}-i \mu \omega a_{2}-i \frac{\omega \beta_{22}}{2} a_{1}\left(\cos \phi_{2}-i \sin \phi_{2}\right) \\
& -\frac{\beta_{21}}{2} a_{1}\left(\cos \phi_{2}-i \sin \phi_{2}\right)-\frac{\beta_{5}}{4} a_{1}^{2} a_{2} \\
& -\frac{\beta_{5}}{8} a_{1}^{2} a_{2}\left(\cos \left(2 \phi_{2}\right)-i \sin \left(2 \phi_{2}\right)\right)-\frac{3 \beta_{5}}{8} a_{2}^{3} \\
& +\frac{\beta_{24} f^{2}}{8} a_{2}\left(\cos \left(2 \phi_{1}-2 \phi_{2}\right)+i \sin \left(2 \phi_{1}-2 \phi_{2}\right)\right)+\frac{\beta_{24} f^{2}}{4} a_{2}=0
\end{aligned}
$$

$$
\begin{aligned}
& -i \omega_{1} \dot{a}_{3}+\omega_{1} a_{3} \dot{\delta}_{3}-i \mu_{1} \omega_{1} a_{3}+\frac{c_{3}}{2} a_{1}\left(\cos \left(\phi_{3}+\omega \tau_{1}\right)\right. \\
& \left.-i \sin \left(\phi_{3}+\omega \tau_{1}\right)\right)=0
\end{aligned}
$$

$$
\begin{aligned}
& -i \omega_{2} \dot{a}_{4}+\omega_{2} a_{4} \dot{\delta}_{4}-i \mu_{2} \omega_{2} a_{4}+\frac{c_{4}}{4} a_{1} a_{4}\left(\cos \left(\phi_{4}+\omega \tau_{1}\right)\right. \\
& \left.-i \sin \left(\phi_{4}+\omega \tau_{1}\right)\right)=0
\end{aligned}
$$

$$
\begin{aligned}
& -i \omega_{3} \dot{a}_{5}+\omega_{3} a_{5} \dot{\delta}_{5}-i \mu_{3} \omega_{3} a_{5}+\frac{c_{4}}{4} a_{1} a_{5}\left(\cos \left(\phi_{5}+\omega \tau_{1}\right)\right. \\
& \left.-i \sin \left(\phi_{5}+\omega \tau_{1}\right)\right)=0
\end{aligned}
$$

where $\phi_{1}=\sigma_{1} t-\delta_{1}, \phi_{2}=\delta_{2}-\delta_{1}, \phi_{3}=\sigma_{2} t+\delta_{3}-\delta_{1}$, $\phi_{4}=\sigma_{3} t+2 \delta_{4}-\delta_{1}$, and $\phi_{5}=\sigma_{4} t+2 \delta_{5}-\delta_{1}$. Separating the real and imaginary parts in Eq. B10 along with using the new definitions of $\phi_{n}$ and $\dot{\phi}_{n}$ guide us the Eq. 6.

\section{Appendix C}

$J_{11}=\frac{\partial \dot{a}_{1}}{\partial a_{1}}=-\mu-\frac{\beta_{5}}{8 \omega} a_{2}^{2} \sin \left(2 \phi_{2}\right)+\frac{\beta_{14} f^{2}}{8 \omega} \sin \left(2 \phi_{1}\right)$

$J_{12}=\frac{\partial \dot{a}_{1}}{\partial \phi_{1}}=\frac{\beta_{14} f^{2}}{4 \omega} a_{1} \cos \left(2 \phi_{1}\right)+\frac{\beta_{16} \Omega f}{2 \omega} \sin \phi_{1}$

$J_{13}=\frac{\partial \dot{a}_{1}}{\partial a_{2}}=-\frac{\beta_{13}}{2} \cos \phi_{2}-\frac{\beta_{5}}{4 \omega} a_{1} a_{2} \sin \left(2 \phi_{2}\right)-\frac{\beta_{11}}{2 \omega} \sin \phi_{2}$

$J_{14}=\frac{\partial \dot{a}_{1}}{\partial \phi_{2}}=\frac{\beta_{13}}{2} a_{2} \sin \phi_{2}-\frac{\beta_{5}}{4 \omega} a_{1} a_{2}^{2} \cos \left(2 \phi_{2}\right)-\frac{\beta_{11}}{2 \omega} a_{2} \cos \phi_{2}$

$J_{15}=\frac{\partial \dot{a}_{1}}{\partial a_{3}}=\frac{c_{1}}{2 \omega} \sin \left(\phi_{3}-\omega_{1} \tau_{2}\right)$

$J_{16}=\frac{\partial \dot{a}_{1}}{\partial \phi_{3}}=\frac{c_{1}}{2 \omega} a_{3} \cos \left(\phi_{3}-\omega_{1} \tau_{2}\right)$

$J_{17}=\frac{\partial \dot{a}_{1}}{\partial a_{4}}=\frac{c_{2}}{2 \omega} a_{4} \sin \left(\phi_{4}-2 \omega_{2} \tau_{2}\right)$

$J_{18}=\frac{\partial \dot{a}_{1}}{\partial \phi_{4}}=\frac{c_{2}}{4 \omega} a_{4}^{2} \cos \left(\phi_{4}-2 \omega_{2} \tau_{2}\right)$

$J_{19}=\frac{\partial \dot{a}_{1}}{\partial a_{5}}=\frac{c_{2}}{2 \omega} a_{5} \sin \left(\phi_{5}-2 \omega_{3} \tau_{2}\right)$

$J_{1,10}=\frac{\partial \dot{a}_{1}}{\partial \phi_{5}}=\frac{c_{2}}{4 \omega} a_{5}^{2} \cos \left(\phi_{5}-2 \omega_{3} \tau_{2}\right)$

$J_{21}=\frac{\partial \dot{\phi}_{1}}{\partial a_{1}}=-\frac{\beta_{13}}{2} \frac{a_{2}}{a_{1}^{2}} \sin \phi_{2}+\frac{\beta_{11}}{2 \omega} \frac{a_{2}}{a_{1}^{2}} \cos \phi_{2}-\frac{3 \beta_{5}}{4 \omega} a_{1}$

$-\frac{\beta_{16} \Omega f}{2 \omega} \frac{1}{a_{1}^{2}} \sin \phi_{1}-\frac{c_{1}}{2 \omega} \frac{a_{3}}{a_{1}^{2}} \cos \left(\phi_{3}-\omega_{1} \tau_{2}\right)$

$-\frac{c_{2}}{4 \omega} \frac{a_{4}^{2}}{a_{1}^{2}} \cos \left(\phi_{4}-2 \omega_{2} \tau_{2}\right)-\frac{c_{2}}{4 \omega} \frac{a_{5}^{2}}{a_{1}^{2}} \cos \left(\phi_{5}-2 \omega_{3} \tau_{2}\right)$

$J_{22}=\frac{\partial \dot{\phi}_{1}}{\partial \phi_{1}}=-\frac{\beta_{14} f^{2}}{4 \omega} \sin \left(2 \phi_{1}\right)+\frac{\beta_{16} \Omega f}{2 \omega} \frac{1}{a_{1}} \cos \phi_{1}$

$J_{23}=\frac{\partial \dot{\phi}_{1}}{\partial a_{2}}=\frac{\beta_{13}}{2} \frac{1}{a_{1}} \sin \phi_{2}-\frac{\beta_{5}}{4 \omega} a_{2} \cos \left(2 \phi_{2}\right)$

$-\frac{\beta_{11}}{2 \omega} \frac{1}{a_{1}} \cos \phi_{2}-\frac{\beta_{5}}{2 \omega} a_{2}$ 


$$
\begin{aligned}
& J_{24}=\frac{\partial \dot{\phi}_{1}}{\partial \phi_{2}}=\frac{\beta_{13}}{2} \frac{a_{2}}{a_{1}} \cos \phi_{2}+\frac{\beta_{5}}{4 \omega} a_{2}^{2} \sin \left(2 \phi_{2}\right)+\frac{\beta_{11}}{2 \omega} \frac{a_{2}}{a_{1}} \sin \phi_{2} \quad J_{42}=\frac{\partial \dot{\phi}_{2}}{\partial \phi_{1}}=\frac{\beta_{24} f^{2}}{4 \omega} \sin \left(2 \phi_{1}-2 \phi_{2}\right)-\frac{\beta_{14} f^{2}}{4 \omega} \sin \left(2 \phi_{1}\right) \\
& J_{25}=\frac{\partial \dot{\phi}_{1}}{\partial a_{3}}=\frac{c_{1}}{2 \omega} \frac{1}{a_{1}} \cos \left(\phi_{3}-\omega_{1} \tau_{2}\right) \\
& +\frac{\beta_{16} \Omega f}{2 \omega} \frac{1}{a_{1}} \cos \phi_{1} \\
& J_{26}=\frac{\partial \dot{\phi}_{1}}{\partial \phi_{3}}=\frac{c_{1}}{2 \omega} \frac{a_{3}}{a_{1}} \sin \left(\phi_{3}-\omega_{1} \tau_{2}\right) \\
& J_{43}=\frac{\partial \dot{\phi}_{2}}{\partial a_{2}}=-\frac{\beta_{22}}{2} \frac{a_{1}}{a_{2}^{2}} \sin \phi_{2}-\frac{\beta_{21}}{2 \omega} \frac{a_{1}}{a_{2}^{2}} \cos \phi_{2}+\frac{3 \beta_{5}}{4 \omega} a_{2} \\
& +\frac{\beta_{13}}{2} \frac{1}{a_{1}} \sin \phi_{2}-\frac{\beta_{5}}{4 \omega} a_{2} \cos \left(2 \phi_{2}\right)-\frac{\beta_{11}}{2 \omega} \frac{1}{a_{1}} \cos \phi_{2}-\frac{\beta_{5}}{2 \omega} a_{2} \\
& J_{27}=\frac{\partial \dot{\phi}_{1}}{\partial a_{4}}=\frac{c_{2}}{2 \omega} \frac{a_{4}}{a_{1}} \cos \left(\phi_{4}-2 \omega_{2} \tau_{2}\right) \\
& J_{28}=\frac{\partial \dot{\phi}_{1}}{\partial \phi_{4}}=-\frac{c_{2}}{4 \omega} \frac{a_{4}^{2}}{a_{1}} \sin \left(\phi_{4}-2 \omega_{2} \tau_{2}\right) \\
& J_{29}=\frac{\partial \dot{\phi}_{1}}{\partial a_{5}}=\frac{c_{2}}{2 \omega} \frac{a_{5}}{a_{1}} \cos \left(\phi_{5}-2 \omega_{3} \tau_{2}\right) \\
& J_{2,10}=\frac{\partial \dot{\phi}_{1}}{\partial \phi_{5}}=-\frac{c_{2}}{4 \omega} \frac{a_{5}^{2}}{a_{1}} \sin \left(\phi_{5}-2 \omega_{3} \tau_{2}\right) \\
& J_{31}=\frac{\partial \dot{a}_{2}}{\partial a_{2}}=-\frac{\beta_{22}}{2} \cos \phi_{2}+\frac{\beta_{5}}{4 \omega} a_{1} a_{2} \sin \left(2 \phi_{2}\right)+\frac{\beta_{21}}{2 \omega} \sin \phi_{2} \\
& J_{44}=\frac{\partial \dot{\phi}_{2}}{\partial \phi_{2}}=\frac{\beta_{22}}{2} \frac{a_{1}}{a_{2}} \cos \phi_{2}-\frac{\beta_{5}}{4 \omega} a_{1}^{2} \sin \left(2 \phi_{2}\right) \\
& -\frac{\beta_{24} f^{2}}{4 \omega} \sin \left(2 \phi_{1}-2 \phi_{2}\right)-\frac{\beta_{21}}{2 \omega} \frac{a_{1}}{a_{2}} \sin \phi_{2} \\
& +\frac{\beta_{13}}{2} \frac{a_{2}}{a_{1}} \cos \phi_{2}+\frac{\beta_{5}}{4 \omega} a_{2}^{2} \sin \left(2 \phi_{2}\right)+\frac{\beta_{11}}{2 \omega} \frac{a_{2}}{a_{1}} \sin \phi_{2} \\
& J_{45}=\frac{\partial \dot{\phi}_{2}}{\partial a_{3}}=\frac{c_{1}}{2 \omega} \frac{1}{a_{1}} \cos \left(\phi_{3}-\omega_{1} \tau_{2}\right) \\
& J_{46}=\frac{\partial \dot{\phi}_{2}}{\partial \phi_{3}}=-\frac{c_{1}}{2 \omega} \frac{a_{3}}{a_{1}} \sin \left(\phi_{3}-\omega_{1} \tau_{2}\right) \\
& J_{47}=\frac{\partial \dot{\phi}_{2}}{\partial a_{4}}=\frac{c_{2}}{2 \omega} \frac{a_{4}}{a_{1}} \cos \left(\phi_{4}-2 \omega_{2} \tau_{2}\right) \\
& J_{32}=\frac{\partial \dot{a}_{2}}{\partial \phi_{1}}=\frac{\beta_{24} f^{2}}{4 \omega} a_{2} \cos \left(2 \phi_{1}-2 \phi_{2}\right) \\
& J_{48}=\frac{\partial \dot{\phi}_{2}}{\partial \phi_{4}}=-\frac{c_{2}}{4 \omega} \frac{a_{4}^{2}}{a_{1}} \sin \left(\phi_{4}-2 \omega_{2} \tau_{2}\right) \\
& J_{33}=\frac{\partial \dot{a}_{2}}{\partial a_{2}}=-\mu+\frac{\beta_{5}}{8 \omega} a_{1}^{2} \sin \left(2 \phi_{2}\right)+\frac{\beta_{24} f^{2}}{8 \omega} \sin \left(2 \phi_{1}-2 \phi_{2}\right) \\
& J_{49}=\frac{\partial \dot{\phi}_{2}}{\partial a_{5}}=\frac{c_{2}}{2 \omega} \frac{a_{5}}{a_{1}} \cos \left(\phi_{5}-2 \omega_{3} \tau_{2}\right) \\
& J_{34}=\frac{\partial \dot{a}_{2}}{\partial \phi_{2}}=\frac{\beta_{22}}{2} a_{1} \sin \phi_{2}+\frac{\beta_{5}}{4 \omega} a_{1}^{2} a_{2} \cos \left(2 \phi_{2}\right) \\
& -\frac{\beta_{24} f^{2}}{4 \omega} a_{2} \cos \left(2 \phi_{1}-2 \phi_{2}\right)+\frac{\beta_{21}}{2 \omega} a_{1} \cos \phi_{2} \\
& J_{35}=J_{36}=J_{37}=J_{38}=J_{39}=J_{3,10}=0 \\
& J_{4,10}=\frac{\partial \dot{\phi}_{2}}{\partial \phi_{5}}=-\frac{c_{2}}{4 \omega} \frac{a_{5}^{2}}{a_{1}} \sin \left(\phi_{5}-2 \omega_{3} \tau_{2}\right) \\
& J_{51}=\frac{\partial \dot{a}_{3}}{\partial a_{1}}=-\frac{c_{3}}{2 \omega_{1}} \sin \left(\phi_{3}+\omega \tau_{1}\right) \\
& J_{41}=\frac{\partial \dot{\phi}_{2}}{\partial a_{1}}=\frac{\beta_{22}}{2} \frac{1}{a_{2}} \sin \phi_{2}+\frac{\beta_{21}}{2 \omega} \frac{1}{a_{2}} \cos \phi_{2}+\frac{\beta_{5}}{2 \omega} a_{1} \\
& J_{52}=J_{53}=J_{54}=J_{57}=J_{58}=J_{59}=J_{5,10}=0 \\
& +\frac{\beta_{5}}{4 \omega} a_{1} \cos \left(2 \phi_{2}\right)-\frac{3 \beta_{5}}{4 \omega} a_{1}-\frac{\beta_{13}}{2} \frac{a_{2}}{a_{1}^{2}} \sin \phi_{2} \\
& +\frac{\beta_{11}}{2 \omega} \frac{a_{2}}{a_{1}^{2}} \cos \phi_{2}-\frac{\beta_{16} \Omega f}{2 \omega} \frac{1}{a_{1}^{2}} \sin \phi_{1}-\frac{c_{1}}{2 \omega} \frac{a_{3}}{a_{1}^{2}} \cos \left(\phi_{3}-\omega_{1} \tau_{2}\right) \\
& -\frac{c_{2}}{4 \omega} \frac{a_{4}^{2}}{a_{1}^{2}} \cos \left(\phi_{4}-2 \omega_{2} \tau_{2}\right)-\frac{c_{2}}{4 \omega} \frac{a_{5}^{2}}{a_{1}^{2}} \cos \left(\phi_{5}-2 \omega_{3} \tau_{2}\right) \\
& J_{55}=\frac{\partial \dot{a}_{3}}{\partial a_{3}}=-\mu_{1} \\
& J_{56}=\frac{\partial \dot{a}_{3}}{\partial \phi_{3}}=-\frac{c_{3}}{2 \omega_{1}} a_{1} \cos \left(\phi_{3}+\omega \tau_{1}\right)
\end{aligned}
$$




$$
\begin{aligned}
& J_{61}=\frac{\partial \dot{\phi}_{3}}{\partial a_{1}}=-\frac{c_{3}}{2 \omega_{1}} \frac{1}{a_{3}} \cos \left(\phi_{3}+\omega \tau_{1}\right)-\frac{\beta_{13}}{2} \frac{a_{2}}{a_{1}^{2}} \sin \phi_{2} \\
& +\frac{\beta_{11}}{2 \omega} \frac{a_{2}}{a_{1}^{2}} \cos \phi_{2}-\frac{3 \beta_{5}}{4 \omega} a_{1}-\frac{\beta_{16} \Omega f}{2 \omega} \frac{1}{a_{1}^{2}} \sin \phi_{1} \\
& -\frac{c_{1}}{2 \omega} \frac{a_{3}}{a_{1}^{2}} \cos \left(\phi_{3}-\omega_{1} \tau_{2}\right)-\frac{c_{2}}{4 \omega} \frac{a_{4}^{2}}{a_{1}^{2}} \cos \left(\phi_{4}-2 \omega_{2} \tau_{2}\right) \\
& -\frac{c_{2}}{4 \omega} \frac{a_{5}^{2}}{a_{1}^{2}} \cos \left(\phi_{5}-2 \omega_{3} \tau_{2}\right) \\
& J_{62}=\frac{\partial \dot{\phi}_{3}}{\partial \phi_{1}}=-\frac{\beta_{14} f^{2}}{4 \omega} \sin \left(2 \phi_{1}\right)+\frac{\beta_{16} \Omega f}{2 \omega} \frac{1}{a_{1}} \cos \phi_{1} \\
& J_{63}=\frac{\partial \dot{\phi}_{3}}{\partial a_{2}}=\frac{\beta_{13}}{2} \frac{1}{a_{1}} \sin \phi_{2}-\frac{\beta_{5}}{4 \omega} a_{2} \cos \left(2 \phi_{2}\right) \\
& -\frac{\beta_{11}}{2 \omega} \frac{1}{a_{1}} \cos \phi_{2}-\frac{\beta_{5}}{2 \omega} a_{2} \\
& J_{66}=\frac{\partial \dot{\phi}_{3}}{\partial \phi_{3}}=\frac{c_{3}}{2 \omega_{1}} \frac{a_{1}}{a_{3}} \sin \left(\phi_{3}+\omega \tau_{1}\right)-\frac{c_{1}}{2 \omega} \frac{a_{3}}{a_{1}} \sin \left(\phi_{3}-\omega_{1} \tau_{2}\right) \\
& J_{68}=\frac{\partial \dot{\phi}_{3}}{\partial \phi_{4}}=-\frac{c_{2}}{4 \omega} \frac{a_{4}^{2}}{a_{1}} \sin \left(\phi_{4}-2 \omega_{2} \tau_{2}\right) \\
& J_{69}=\frac{\partial \dot{\phi}_{3}}{\partial a_{5}}=\frac{c_{2}}{2 \omega} \frac{a_{5}}{a_{1}} \cos \left(\phi_{5}-2 \omega_{3} \tau_{2}\right) \\
& J_{6,10}=\frac{\partial \dot{\phi}_{3}}{\partial \phi_{5}}=-\frac{c_{2}}{4 \omega} \frac{a_{5}^{2}}{a_{1}} \sin \left(\phi_{5}-2 \omega_{3} \tau_{2}\right) \\
& J_{71}=\frac{\partial \dot{a}_{4}}{\partial a_{1}}=-\frac{c_{4}}{4 \omega_{2}} a_{4} \sin \left(\phi_{4}+\omega \tau_{1}\right) \\
& J_{72}=J_{73}=J_{74}=J_{75}=J_{76}=J_{79}=J_{7,10}=0 \\
& J_{77}=\frac{\partial \dot{a}_{4}}{\partial a_{4}}=-\mu_{2}-\frac{c_{4}}{4 \omega_{2}} a_{1} \sin \left(\phi_{4}+\omega \tau_{1}\right) \\
& J_{78}=\frac{\partial \dot{a}_{4}}{\partial \phi_{4}}=-\frac{c_{4}}{4 \omega_{2}} a_{1} a_{4} \cos \left(\phi_{4}+\omega \tau_{1}\right) \\
& \begin{aligned}
J_{81}= & \frac{\partial \dot{\phi}_{4}}{\partial a_{1}}=-\frac{c_{4}}{2 \omega_{2}} \cos \left(\phi_{4}+\omega \tau_{1}\right)-\frac{\beta_{13}}{2} \frac{a_{2}}{a_{1}^{2}} \sin \phi_{2} \\
& +\frac{\beta_{11}}{2 \omega} \frac{a_{2}}{a_{1}^{2}} \cos \phi_{2}-\frac{3 \beta_{5}}{4 \omega} a_{1}-\frac{\beta_{16} \Omega f}{2 \omega} \frac{1}{a_{1}^{2}} \sin \phi_{1} \\
& -\frac{c_{1}}{2 \omega} \frac{a_{3}}{a_{1}^{2}} \cos \left(\phi_{3}-\omega_{1} \tau_{2}\right)-\frac{c_{2}}{4 \omega} \frac{a_{4}^{2}}{a_{1}^{2}} \cos \left(\phi_{4}-2 \omega_{2} \tau_{2}\right) \\
& -\frac{c_{2}}{4 \omega} \frac{a_{5}^{2}}{a_{1}^{2}} \cos \left(\phi_{5}-2 \omega_{3} \tau_{2}\right)
\end{aligned} \\
& J_{82}=\frac{\partial \dot{\phi}_{4}}{\partial \phi_{1}}=-\frac{\beta_{14} f^{2}}{4 \omega} \sin \left(2 \phi_{1}\right)+\frac{\beta_{16} \Omega f}{2 \omega} \frac{1}{a_{1}} \cos \phi_{1} \\
& J_{83}=\frac{\partial \dot{\phi}_{4}}{\partial a_{2}}=\frac{\beta_{13}}{2} \frac{1}{a_{1}} \sin \phi_{2}-\frac{\beta_{11}}{2 \omega} \frac{1}{a_{1}} \cos \phi_{2} \\
& -\frac{\beta_{5}}{2 \omega} a_{2}-\frac{\beta_{5}}{4 \omega} a_{2} \cos \left(2 \phi_{2}\right) \\
& J_{84}=\frac{\partial \dot{\phi}_{4}}{\partial \phi_{2}}=\frac{\beta_{13}}{2} \frac{a_{2}}{a_{1}} \cos \phi_{2}+\frac{\beta_{11}}{2 \omega} \frac{a_{2}}{a_{1}} \sin \phi_{2}+\frac{\beta_{5}}{4 \omega} a_{2}^{2} \sin \left(2 \phi_{2}\right) \\
& J_{85}=\frac{\partial \dot{\phi}_{4}}{\partial a_{3}}=\frac{c_{1}}{2 \omega} \frac{1}{a_{1}} \cos \left(\phi_{3}-\omega_{1} \tau_{2}\right) \\
& J_{86}=\frac{\partial \dot{\phi}_{4}}{\partial \phi_{3}}=-\frac{c_{1}}{2 \omega} \frac{a_{3}}{a_{1}} \sin \left(\phi_{3}-\omega_{1} \tau_{2}\right) \\
& J_{87}=\frac{\partial \dot{\phi}_{4}}{\partial a_{4}}=\frac{c_{2}}{2 \omega} \frac{a_{4}}{a_{1}} \cos \left(\phi_{4}-2 \omega_{2} \tau_{2}\right) \\
& J_{88}=\frac{\partial \dot{\phi}_{4}}{\partial \phi_{4}}=\frac{c_{4}}{2 \omega_{2}} a_{1} \sin \left(\phi_{4}+\omega \tau_{1}\right)-\frac{c_{2}}{4 \omega} \frac{a_{4}^{2}}{a_{1}} \sin \left(\phi_{4}-2 \omega_{2} \tau_{2}\right) \\
& J_{89}=\frac{\partial \dot{\phi}_{4}}{\partial a_{5}}=\frac{c_{2}}{2 \omega} \frac{a_{5}}{a_{1}} \cos \left(\phi_{5}-2 \omega_{3} \tau_{2}\right) \\
& J_{8,10}=\frac{\partial \dot{\phi}_{4}}{\partial \phi_{5}}=-\frac{c_{2}}{4 \omega} \frac{a_{5}^{2}}{a_{1}} \sin \left(\phi_{5}-2 \omega_{3} \tau_{2}\right) \\
& J_{91}=\frac{\partial \dot{a}_{5}}{\partial a_{1}}=-\frac{c_{4}}{4 \omega_{3}} a_{5} \sin \left(\phi_{5}+\omega \tau_{1}\right) \\
& J_{92}=J_{93}=J_{94}=J_{95}=J_{96}=J_{97}=J_{98}=0 \\
& J_{99}=\frac{\partial \dot{a}_{5}}{\partial a_{5}}=-\mu_{3}-\frac{c_{4}}{4 \omega_{3}} a_{1} \sin \left(\phi_{5}+\omega \tau_{1}\right)
\end{aligned}
$$




$$
\begin{aligned}
& J_{9,10}=\frac{\partial \dot{a}_{5}}{\partial \phi_{5}}=-\frac{c_{4}}{4 \omega_{3}} a_{1} a_{5} \cos \left(\phi_{5}+\omega \tau_{1}\right) \\
& J_{10,1}=\frac{\partial \dot{\phi}_{5}}{\partial a_{1}}=-\frac{c_{4}}{2 \omega_{3}} \cos \left(\phi_{5}+\omega \tau_{1}\right)-\frac{\beta_{13}}{2} \frac{a_{2}}{a_{1}^{2}} \sin \phi_{2} \\
& +\frac{\beta_{11}}{2 \omega} \frac{a_{2}}{a_{1}^{2}} \cos \phi_{2}-\frac{3 \beta_{5}}{4 \omega} a_{1}-\frac{\beta_{16} \Omega f}{2 \omega} \frac{1}{a_{1}^{2}} \sin \phi_{1} \\
& -\frac{c_{1}}{2 \omega} \frac{a_{3}}{a_{1}^{2}} \cos \left(\phi_{3}-\omega_{1} \tau_{2}\right)-\frac{c_{2}}{4 \omega} \frac{a_{4}^{2}}{a_{1}^{2}} \cos \left(\phi_{4}-2 \omega_{2} \tau_{2}\right) \\
& -\frac{c_{2}}{4 \omega} \frac{a_{5}^{2}}{a_{1}^{2}} \cos \left(\phi_{5}-2 \omega_{3} \tau_{2}\right) \\
& J_{10,2}=\frac{\partial \dot{\phi}_{5}}{\partial \phi_{1}}=-\frac{\beta_{14} f^{2}}{4 \omega} \sin \left(2 \phi_{1}\right)+\frac{\beta_{16} \Omega f}{2 \omega} \frac{1}{a_{1}} \cos \phi_{1} \\
& J_{10,3}=\frac{\partial \dot{\phi}_{5}}{\partial a_{2}}=\frac{\beta_{13}}{2} \frac{1}{a_{1}} \sin \phi_{2}-\frac{\beta_{11}}{2 \omega} \frac{1}{a_{1}} \cos \phi_{2} \\
& -\frac{\beta_{5}}{2 \omega} a_{2}-\frac{\beta_{5}}{4 \omega} a_{2} \cos \left(2 \phi_{2}\right) \\
& J_{10,4}=\frac{\partial \dot{\phi}_{5}}{\partial \phi_{2}}=\frac{\beta_{13}}{2} \frac{a_{2}}{a_{1}} \cos \phi_{2}+\frac{\beta_{11}}{2 \omega} \frac{a_{2}}{a_{1}} \sin \phi_{2}+\frac{\beta_{5}}{4 \omega} a_{2}^{2} \sin \left(2 \phi_{2}\right) \\
& J_{10,5}=\frac{\partial \dot{\phi}_{5}}{\partial a_{3}}=\frac{c_{1}}{2 \omega} \frac{1}{a_{1}} \cos \left(\phi_{3}-\omega_{1} \tau_{2}\right) \\
& J_{10,6}=\frac{\partial \dot{\phi}_{5}}{\partial \phi_{3}}=-\frac{c_{1}}{2 \omega} \frac{a_{3}}{a_{1}} \sin \left(\phi_{3}-\omega_{1} \tau_{2}\right) \\
& J_{10,7}=\frac{\partial \dot{\phi}_{5}}{\partial a_{4}}=\frac{c_{2}}{2 \omega} \frac{a_{4}}{a_{1}} \cos \left(\phi_{4}-2 \omega_{2} \tau_{2}\right) \\
& J_{10,8}=\frac{\partial \dot{\phi}_{5}}{\partial \phi_{4}}=-\frac{c_{2}}{4 \omega} \frac{a_{4}^{2}}{a_{1}} \sin \left(\phi_{4}-2 \omega_{2} \tau_{2}\right) \\
& J_{10,9}=\frac{\partial \dot{\phi}_{5}}{\partial a_{5}}=\frac{c_{2}}{2 \omega} \frac{a_{5}}{a_{1}} \cos \left(\phi_{5}-2 \omega_{3} \tau_{2}\right) \\
& J_{10,10}=\frac{\partial \dot{\phi}_{5}}{\partial \phi_{5}}=\frac{c_{4}}{2 \omega_{3}} a_{1} \sin \left(\phi_{5}+\omega \tau_{1}\right)-\frac{c_{2}}{4 \omega} \frac{a_{5}^{2}}{a_{1}} \sin \left(\phi_{5}-2 \omega_{3} \tau_{2}\right)
\end{aligned}
$$

\section{References}

1. Yoo HH, Kwak JY, Chung J (2001) Vibration analysis of rotating pre-twisted blades with a concentrated mass. J Sound Vib 240:891-908
2. Sinha SK (2004) Dynamic characteristics of a flexible bladed rotor with Coulomb damping due to tip-rub. J Sound Vib 273:875-919

3. Fazelzadeh SA, Malekzadeh P, Zahedinejad P, Hosseini M (2007) Vibration analysis of functionally graded thinwalled rotating blades under high temperature supersonic flow using the differential quadrature method. J Sound Vib 306:333-348

4. Yao MH, Chen YP, Zhang W (2012) Nonlinear vibrations of blade with varying rotating speed. Nonlinear Dyn 68:487-504

5. Yao MH, Zhang W, Chen YP (2014) Analysis on nonlinear oscillations and resonant responses of a compressor blade. Acta Mech 225:3483-3510

6. Younesian D, Esmailzadeh E (2011) Vibration suppression of rotating beams using time-varying internal tensile force. J Sound Vib 330:308-320

7. Park J-S, Kim J-H (2005) Analytical development of single crystal Macro Fiber Composite actuators for active twist rotor blades. Smart Mater Struct 14:745-753

8. Choi S-C, Park J-S, Kim J-H (2006) Active damping of rotating composite thin-walled beams using MFC actuators and PVDF sensors. Compos Struct 76:362-374

9. Choi S-C, Park J-S, Kim J-H (2007) Vibration control of pre-twisted rotating composite thin-walled beams with piezoelectric fiber composites. J Sound Vib 300:176-196

10. Vadiraja DN, Sahasrabudhe AD (2009) Vibration analysis and optimal control of rotating pre-twisted thin-walled beams using MFC actuators and sensors. Thin-Walled Struct 47:555-567

11. Warminski J, Bochenski M, Jarzyna W, Filipek $P$, Augustyniak $M$ (2011) Active suppression of nonlinear composite beam vibrations by selected control algorithms. Commun Nonlinear Sci Numer Simulat 16:2237-2248

12. El-Ganaini WA, Saeed NA, Eissa M (2013) Positive position feedback controller (PPF) for suppression of nonlinear system vibration. Nonlinear Dyn 72:517-537

13. Kamel M, Kandil A, El-Ganaini WA, Eissa M (2014) Active vibration control of a nonlinear magnetic levitation system via nonlinear saturation controller (NSC). Nonlinear Dyn 77:605-619

14. Kandil A, El-Gohary H (2016) Suppressing the nonlinear vibrations of a compressor blade via a nonlinear saturation controller. J Vibrat Control. https://doi.org/10.1177/1077546316661680

15. Zhao YY, Xu J (2007) Effects of delayed feedback control on nonlinear vibration absorber system. J Sound Vib 308:212-230

16. Eissa M, Kandil A, El-Ganaini WA, Kamel M (2015) Vibration suppression of a nonlinear magnetic levitation system via time delayed nonlinear saturation controller. Int J Non-Linear Mech 72:23-41

17. El-Ganaini WA, Kandil A, Eissa M, Kamel M (2016) Effects of delayed time active controller on the vibration of a nonlinear magnetic levitation system to multi excitations. J Vib Control 22(5):1257-1275

18. Kandil A, Eissa M (2017) Improvement of positive position feedback controller for suppressing compressor blade oscillations. Nonlinear Dyn 90:1727-1753

19. Kandil A, El-Ganaini WA (2018) Investigation of the time delay effect on the control of rotating blade vibrations. Eur J Mech A Solids 72:16-40

20. Nayfeh AH, Mook DT (1995) Nonlinear Oscillations. Wiley, New York

Publisher's Note Springer Nature remains neutral with regard to jurisdictional claims in published maps and institutional affiliations. 